\title{
Nonlinear variational surface waves
}

\author{
Lawrence Austria and John K. Hunter*
}

\begin{abstract}
We derive nonlocal asymptotic equations for weakly nonlinear surface wave solutions of variational wave equations in a half-space. These equations are analogous to, but different from, equations that describe weakly nonlinear Rayleigh waves in elasticity and other hyperbolic conservation laws. We prove short time existence of smooth solutions of a simplified, but representative, asymptotic equation and present numerical solutions which show the formation of cusp-singularities. This singularity formation on the boundary is a different mechanism for the nonlinear breakdown of smooth solutions of hyperbolic IBVPs from the more familiar one of singularity formation in the interior.
\end{abstract}

\section{Introduction}

In this paper, we consider initial-boundary value problems (IBVPs) in a half-space $\mathbb{R}_{+}^{d}$ for systems of wave equations for $u: \mathbb{R}_{+}^{d} \times \mathbb{R} \rightarrow \mathbb{R}^{n}$ that arise from variational principles of the form

$$
\delta \int_{\mathbb{R}_{+}^{d} \times \mathbb{R}}\left\{\frac{1}{2}\left|u_{t}\right|^{2}-W(u, \nabla u)\right\} d x d t=0,
$$

where the potential energy density $W(u, \nabla u)$ is a quadratic function of $\nabla u$ with coefficients depending on $u$.

The nonlinearity in the resulting variational, but non-conservative, wave equations differs qualitatively from the nonlinearity in conservative quasilinear wave equations, such as nonlinear elasticity, where $W(\nabla u)$ is independent of $u$ but not quadratic in $\nabla u$. We are interested in comparing and contrasting the effects of nonlinearity on these types of waves.

We study IBVPs that are stable but not uniformly stable, which occurs when the IBVP has surface wave solutions. We focus on problems with "genuine" or "finite-energy" surface waves that propagate along the boundary and decay exponentially into the interior, rather than "radiative" surface

\footnotetext{
*Supported by the NSF under Grant No. DMS-1312342.
} 
Table 1: Model asymptotic equations for weakly nonlinear bulk and finiteenergy surface waves with amplitude function $u(x, t)$ governed by hyperbolic conservation laws and variational principles (1). In this table, $H$ denotes the Hilbert transform with respect to $x$, and $h=H[u]$. The bulk-wave equations are the inviscid Burgers equation and the HS equation; the surface-wave equations are the HIZ equation and the equation derived in this paper

\begin{tabular}{|c|c|c|}
\hline & Conservation Laws & Variational Equations \\
\hline Bulk & $u_{t}+\left(\frac{1}{2} u^{2}\right)_{x}=0$ & {$\left[u_{t}+\left(\frac{1}{2} u^{2}\right)_{x}\right]_{x}=\frac{1}{2} u_{x}^{2}$} \\
\hline Surface & $u_{t}+H\left[h h_{x}\right]_{x}+h u_{x x}=0$ & $u_{t}+\left(\frac{1}{2} u^{2}\right)_{x}=H\left[u h_{x}\right]$ \\
\hline
\end{tabular}

waves that are coupled with bulk waves in the interior. The aim of this paper is to derive asymptotic equations for weakly nonlinear, variational genuine surface waves and, in particular, to show that singularities form in these waves on the boundary.

Table 1 summarizes various model asymptotic equations for hyperbolic bulk and surface waves in conservation laws and variational wave equations. We discuss the origin and properties of these equations in greater detail in the remainder of this introduction.

One example of a variational system of the form (1) arises as a description of orientation waves in a massive director field [2, 19]. The orientation of the director is described by a unit vector field

$$
\mathbf{n}: \mathbb{R}_{+}^{3} \times \mathbb{R} \rightarrow \mathbb{S}^{2}
$$

The director field satisfies

$$
\delta \int_{\mathbb{R}_{+}^{3} \times \mathbb{R}}\left\{\frac{1}{2}\left|\mathbf{n}_{t}\right|^{2}-W(\mathbf{n}, \nabla \mathbf{n})\right\} d \mathbf{x} d t=0, \quad \mathbf{n} \cdot \mathbf{n}=1,
$$

where $W(\mathbf{n}, \nabla \mathbf{n})$ is the Oseen-Frank energy function from the continuum theory of nematic liquid crystals $[12,29]$

$$
\begin{aligned}
W(\mathbf{n}, \nabla \mathbf{n})= & \frac{1}{2} \alpha(\operatorname{div} \mathbf{n})^{2}+\frac{1}{2} \beta(\mathbf{n} \cdot \operatorname{curl} \mathbf{n})^{2}+\frac{1}{2} \gamma|\mathbf{n} \times \operatorname{curl} \mathbf{n}|^{2} \\
& +\frac{1}{2} \eta\left[\operatorname{tr}(\nabla \mathbf{n})^{2}-(\operatorname{div} \mathbf{n})^{2}\right]
\end{aligned}
$$


Here, $\alpha, \beta, \gamma, \eta$ are positive constants.

The potential energy density in (3) has a natural geometric origin: $W(\mathbf{n}, \nabla \mathbf{n})$ is the most general function that is quadratic in $\nabla \mathbf{n}$ and invariant under simultaneous orthogonal transformations $\mathbf{x} \mapsto Q \mathbf{x}$ and $\mathbf{n} \mapsto Q \mathbf{n}$ of the independent and dependent variables for all $Q \in S O(3)$. This symmetry is less restrictive than the harmonic map symmetry $\mathbf{x} \mapsto Q \mathbf{x}$ and $\mathbf{n} \mapsto Q^{\prime} \mathbf{n}$ for all $Q, Q^{\prime} \in S O(3)$, which implies that $W$ is proportional to $|\nabla \mathbf{n}|^{2}$, and it allows the coefficients of the quadratic function of $\nabla \mathbf{n}$ to depend on $\mathbf{n}$. The term proportional to $\eta$ in (3) is a null Lagrangian that corresponds to a surface energy term; it influences only the natural boundary conditions.

There has been extensive analysis of the initial value problem for (2)(3) in one space dimension, including a proof of the existence of global weak solutions under suitable assumptions (see $[10,32]$ and the references cited there). In addition, an almost global existence result for smooth, planar solutions in three space dimensions, without boundaries, is proved in [14]. A general existence theory for weak solutions of multi-dimensional variational wave equations is completely open, just as it is for multi-dimensional hyperbolic conservation laws.

A specific motivation for the problems studied in this paper is an analogy between the director-field system (2)-(3) and nonlinear elasticity. The director-field system has two types of bulk waves, called splay and twist waves [2], which are analogous to longitudinal $p$-waves and transverse $s$-waves, respectively, in elasticity. It is interesting to compare the nonlinear behavior of longitudinal, transverse, and surface waves in these two systems.

The deformation gradient of a weakly nonlinear elastic $p$-wave satisfies a quadratically nonlinear inviscid Burgers equation,

$$
u_{t}+\left(\frac{1}{2} u^{2}\right)_{x}=0 .
$$

Nonlinearity leads to the formation of shocks, and after that smooth solutions may be continued by unique weak entropy solutions [11]. On the other hand, the orientation angle of a weakly nonlinear splay wave in a director field satisfies the Hunter-Saxton (HS) equation [19],

$$
\left[u_{t}+\left(\frac{1}{2} u^{2}\right)_{x}\right]_{x}=\frac{1}{2} u_{x}^{2} .
$$

Nonlinearity causes the derivative of solutions to blow up, and after that the solutions may be continued by different classes of continuous weak solutions, 
including ones that conserve energy as well as ones that dissipate energy [22]. Furthermore, the HS equation is completely integrable [21].

Nonlinear effects on small-amplitude transverse waves are weaker. The deformation gradient of an $s$-waves in an isotropic elastic solid satisfies a modified inviscid Burgers equation with a cubic flux [13], while the orientation angle of a twist wave in a director field satisfies a cubically nonlinear equation that is related to the HS equation [2].

An elastic half-space supports Rayleigh surface waves whose energy is localized near the boundary. The bulk $p$ and $s$ wave fields in a linear Rayleigh wave are coupled together through natural, stress-free boundary conditions. Furthermore, as a result of the nonlinear self-interaction of the bulk waves in the interior of the half-space, the displacement of the boundary in a weakly nonlinear Rayleigh wave satisfies a quadratically nonlinear, spatially nonlocal asymptotic equation [16].

A model asymptotic equation for weakly nonlinear Rayleigh waves is

$$
u_{t}+H\left[h h_{x}\right]_{x}+h u_{x x}=0, \quad h=H[u] .
$$

In (5) and below, $H$ denotes the spatial Hilbert transform, which is the linear singular-integral operator defined by

$$
H\left[e^{i k x}\right]=-i(\operatorname{sgn} k) e^{i k x}, \quad \operatorname{sgn} k= \begin{cases}1 & \text { if } k>0 \\ 0 & \text { if } k=0 \\ -1 & \text { if } k<0\end{cases}
$$

Equation (5) was introduced by Hamilton, Il'insky, and Zabolotskaya [15] as a simplification of the full asymptotic equation for Rayleigh waves, so we refer to it as the HIZ equation; it also describes surface waves on a tangential discontinuity in MHD [1]. Thus, (5) serves as a model equation for genuine surface wave solutions of hyperbolic conservation laws, analogous to the inviscid Burger's equation for bulk waves. Other, more complicated, asymptotic surface wave equations arise from hyperbolic conservation laws, but they have the same qualitative scaling and Hamiltonian properties as the HIZ equation [3].

The director-field system (2)-(3) has finite-energy surface wave solutions that are analogous to Rayleigh waves, in which the splay waves and twist waves are linearly coupled through natural boundary conditions and selfinteract nonlinearly in the interior of the half-space. In Section 8, we obtain an asymptotic equation for a weakly nonlinear surface wave in a director field. 
The algebra involved in deriving this equation from the director-field system is extremely complicated. For most of this paper, we therefore analyze a model system of variational wave equations that exhibits the main features in a simpler setting.

Surface waves in IBVPs for scalar wave equations are always radiative (see Majda, p. 144 [24] for an example), and genuine surface waves only arise in systems of wave equations, such as the ones that describe the motion of elastic solids or director fields. As a model, we consider a variational principle for two real-valued functions $u(x, y, t), v(x, y, t)$ defined in the half-space $\mathbb{R}_{+}^{2}=\{(x, y): x>0\}$ of the form

$$
\delta \int_{\mathbb{R}_{+}^{2} \times \mathbb{R}}\left\{\frac{1}{2}\left[u_{t}^{2}+v_{t}^{2}\right]-W(u, v, \nabla u, \nabla v)\right\} d x d y d t=0,
$$

where the potential energy density $W$ is given by

$$
W(u, v, \nabla u, \nabla v)=\frac{1}{2} \alpha^{2}(u)\left[u_{x}^{2}+u_{y}^{2}\right]+\frac{1}{2} \beta^{2}(v)\left[v_{x}^{2}+v_{y}^{2}\right]-\eta\left[u_{x} v_{y}-u_{y} v_{x}\right] .
$$

We assume that the bulk wave speeds $\alpha, \beta: \mathbb{R} \rightarrow \mathbb{R}_{+}$in (8) are smooth, positive functions, and $\eta$ is a real, nonzero constant multiplying the nullLagrangian $u_{x} v_{y}-u_{y} v_{x}$.

The Euler-Lagrange equation for (7)-(8) consists of decoupled variational wave equations in $x>0$,

$$
u_{t t}=\alpha^{2}(u) \Delta u+\alpha(u) \alpha^{\prime}(u)|\nabla u|^{2}, \quad v_{t t}=\beta^{2}(v) \Delta v+\beta(v) \beta^{\prime}(v)|\nabla v|^{2} .
$$

We impose natural boundary conditions on $x=0$,

$$
\alpha^{2}(u) u_{x}-\eta v_{y}=0, \quad \beta^{2}(v) v_{x}+\eta u_{y}=0,
$$

which couple together $u, v$ through the null-Lagrangian.

In Section 2, we derive the Lopatinski condition for the linearization of (9)-(10), which is a necessary condition for the IBVP to be well-posed [6]. Serre [27] gives a full discussion of Lopatinski conditions for wave equations described by variational principles of the form (1). In Section 3, we describe the linearized surface wave solutions, and in Section 4, we use the method of multiple scales to derive an asymptotic equation for weakly nonlinear, genuine surface wave solutions of (9)-(10).

The result is a spectral asymptotic equation of the form

$$
\hat{a}_{\tau}(k, \tau)+i \operatorname{sgn}(k) E_{0} \int_{\mathbb{R}} \Lambda(-k, k-l, l) \hat{a}(k-l, \tau) \hat{a}(l, \tau) d l=0,
$$


where $\hat{a}(k, \tau)$ denotes the amplitude of the surface wave on the boundary as a function of a "slow" time $\tau$ and a tangential wavenumber $k$, and the kernel $\Lambda$ is given by

$$
\begin{aligned}
\Lambda\left(k_{1}, k_{2}, k_{3}\right) & =\left[A_{0}-i B_{0} \operatorname{sgn}\left(k_{1} k_{2} k_{3}\right)\right] \frac{\left|k_{1} k_{2}\right|+\left|k_{2} k_{3}\right|+\left|k_{1} k_{3}\right|}{\left|k_{1}\right|+\left|k_{2}\right|+\left|k_{3}\right|} \\
& -\left[C_{0}-i D_{0} \operatorname{sgn}\left(k_{1} k_{2} k_{3}\right)\right] \frac{k_{1} k_{2}+k_{2} k_{3}+k_{1} k_{3}}{\left|k_{1}\right|+\left|k_{2}\right|+\left|k_{3}\right|} .
\end{aligned}
$$

The constants $A_{0}-E_{0}$ are defined in (29)-(30). For definiteness, we consider solutions on the real line. The same equation applies to spatially periodic solutions after replacing integrals by sums.

The kernel $\Lambda\left(k_{1}, k_{2}, k_{3}\right)$ in (11)-(12) is an interaction coefficient that describes the strength of the quadratically nonlinear interactions between wavenumbers $k_{1}, k_{2}, k_{3}$ that satisfy the three-wave resonance condition

$$
k_{1}+k_{2}+k_{3}=0 .
$$

Only wavenumbers that satisfy this condition appear in (11), and the value of $\Lambda$ on other wavenumbers is irrelevant. It is, however, convenient to retain all three wavenumbers to exhibit the symmetry of $\Lambda\left(k_{1}, k_{2}, k_{3}\right)$. This "detailed balance" symmetry is a consequence of the fact that (11) is Hamiltonian with respect to the complex canonical variables $\hat{a}(k, \tau)$ [3]. In Section 5, we use the Hamiltonian formulation of (7) to derive the same asymptotic equation (11).

In Section 6, we show how to write (11) as a spatial equation for

$$
a(\theta, \tau)=\int \hat{a}(k, \tau) e^{i k \theta} d k,
$$

where $\theta=y-\lambda t$ denotes a spatial variable tangent to the boundary in a reference frame moving with the linearized surface wave speed $\lambda$. We also introduce a simplified asymptotic equation with the kernel

$$
\Lambda\left(k_{1}, k_{2}, k_{3}\right)=\frac{1}{2}\left(\left|k_{1}\right|+\left|k_{2}\right|+\left|k_{3}\right|\right),
$$

corresponding to $A_{0}=C_{0}$ and $B_{0}=D_{0}=0$ in (12). After rescaling $a$ to remove an inessential constant, the associated spatial form of the equation is

$$
a_{\tau}+\left(\frac{1}{2} a^{2}\right)_{\theta}=H[a|\partial| a],
$$


where $H$ is the Hilbert transform with respect to $\theta$, and $|\partial|=H \partial_{\theta}$ has symbol $|k|$.

Equation (14) plays an analogous role for surface wave solutions of variational wave equations to the HIZ equation (5) for hyperbolic conservation laws. It may also provide an example of a nonlocal surface wave equation that is easier to analyze than the HIZ equation.

We remark that the HIZ equation (5) can also be written in the spectral form (11) with a kernel proportional to

$$
\Lambda\left(k_{1}, k_{2}, k_{3}\right)=\frac{2\left|k_{1} k_{2} k_{3}\right|}{\left|k_{1}\right|+\left|k_{2}\right|+\left|k_{3}\right|} .
$$

This kernel is homogeneous of degree two, corresponding to the appearance of two spatial derivatives in (5), whereas the kernel (13) is homogeneous of degree one, corresponding to the appearance of one spatial derivative in (14). This difference in the scaling properties of the interaction coefficient $\Lambda$, when expressed with respect to complex canonical variables, is the fundamental qualitative difference between the weak nonlinearity in surface waves for conservation laws and variational wave equations.

In Section 7, we establish the short-time existence of smooth, spatially periodic solutions of (14) and show some numerical solutions. These simulations indicate that smooth solutions break down in finite time and appear to form cusp singularities proportional to $\operatorname{sgn} \theta|\theta|^{\alpha}$ with $\alpha \approx 1 / 3$, which is in reasonable agreement with an exact time-independent weak cusp solution of (14) with $\alpha=1 / 3$. The corresponding asymptotic solution remains smooth in the interior of the half-space, since its Fourier coefficients with respect to $\theta$ decay exponentially as $|k| \rightarrow \infty$ when $x>0$. Thus, this mechanism for the breakdown of smooth solutions on the boundary differs from the more familiar one of the formation of singularities in the interior, which for variational wave equations is described asymptotically by the HS equation.

It is interesting to compare the singularities in bulk and surface variational waves. Smooth solutions of the HS equation (4) conserve the spatial $L^{2}$-norm of $u_{x}$, and the HS equation has global (dissipative or conservative) weak $H^{1}$-solutions that are Hölder continuous with exponent $1 / 2$ $[7,31]$. In addition, the HS equation has time-independent cusp solutions with $\alpha=2 / 3$. Similarly, one-dimensional variational wave equations of the form (9) have global $H^{1}$-solutions $[8,9]$.

By contrast, smooth solutions of the surface-wave equation (14) conserve the spatial $L^{2}$-norm of $|\partial|^{1 / 2} a$, given in (47), and as noted above the numerical results suggest that general, global weak solutions are Hölder continuous 
with exponent at most approximately equal to $1 / 3$. These weak solutions are less regular than the HS solutions. On the other hand, Sobolev embedding does not explain the fact that they appear to be continuous, since $H^{1 / 2}$-functions need not even be locally bounded. We remark also that the $H^{1 / 2}$-estimate for surface wave solutions on the boundary is consistent with the standard $H^{1}$-energy estimate for free-space solutions of the variational wave equations in (9), since there is a loss of half an $L^{2}$-derivative in the trace map for Sobolev functions,

Finally, in Section 8, we return to an analysis of surface wave solutions of the director-field equations. For definiteness, we consider surface waves on a half-space in the case when the unperturbed director field is tangent to the boundary. The result is an asymptotic equation (70) of the same form as (11), with a more complicated kernel $\Lambda\left(k_{1}, k_{2}, k_{3}\right)$ that is given in (71). Like the model kernel, this kernel is a symmetric, homogeneous function of $\left(k_{1}, k_{2}, k_{3}\right)$ of degree one, and it has an similar relationship to the model kernel as the full Rayleigh-wave kernel has to the HIZ kernel [3, 15].

There are many open problems about nonlocal surface wave equations such as (5) or (14). For example: a proof of singularity formation; the existence of global weak solutions, conservative or dissipative; and whether or not any of these equations are completely integrable. Although these equations are scalar, their intrinsic, and apparently non-removable, nonlocality seems to make their analysis significantly harder than the analysis of the inviscid Burgers or HS equations for bulk waves.

\section{The Lopatinski condition}

The linearization of the model IBVP $(9)-(10)$ at $(u, v)=(0,0)$ consists of a pair of two-dimensional wave equations in the half-space $x>0$,

$$
u_{t t}=\alpha_{0}^{2}\left(u_{x x}+u_{y y}\right), \quad v_{t t}=\beta_{0}^{2}\left(v_{x x}+v_{y y}\right)
$$

where $\alpha_{0}=\alpha(0)$ and $\beta_{0}=\beta(0)$, with the boundary conditions on $x=0$

$$
\alpha_{0}^{2} u_{x}-\eta v_{y}=0, \quad \beta_{0}^{2} v_{x}+\eta u_{y}=0
$$

In this section, we derive the Lopatinski condition for (15)-(16), which is a necessary condition for the well-posedness of the IBVP.

We look for Fourier-Laplace solutions of (15)-(16) of the form

$$
\left[\begin{array}{l}
u \\
v
\end{array}\right]=\left[\begin{array}{l}
\hat{u} e^{-k x} \\
\hat{v} e^{-k x}
\end{array}\right] e^{\tau t+i l y},
$$


where $l \in \mathbb{R}, \tau \in \mathbb{C}$ with $\Re \tau>0$, and $k \in \mathbb{C}$ with $\Re k>0$. Then (17) satisfies (15) if and only if:

$$
\tau^{2}=\alpha_{0}^{2}\left(k^{2}-l^{2}\right), \quad\left[\begin{array}{l}
\hat{u} \\
\hat{v}
\end{array}\right]=\left[\begin{array}{c}
R \\
0
\end{array}\right] ; \quad \text { or } \quad \tau^{2}=\beta_{0}^{2}\left(k^{2}-l^{2}\right), \quad\left[\begin{array}{l}
\hat{u} \\
\hat{v}
\end{array}\right]=\left[\begin{array}{l}
0 \\
S
\end{array}\right] .
$$

The corresponding solution of the PDE for a given tangential wavenumber $l$ and complex frequency $\tau$ is

$$
\left[\begin{array}{l}
u \\
v
\end{array}\right]=\left[\begin{array}{c}
R e^{-k_{\alpha} x} \\
S e^{-k_{\beta} x}
\end{array}\right] e^{\tau t+i l y},
$$

where $R$ and $S$ are arbitrary constants and

$$
k_{\alpha}=\sqrt{l^{2}+\frac{\tau^{2}}{\alpha_{0}^{2}}}, \quad k_{\beta}=\sqrt{l^{2}+\frac{\tau^{2}}{\beta_{0}^{2}}} .
$$

Here, we take the branch of the square root with positive real part, which defines $k_{\alpha}, k_{\beta}$ uniquely in $\Re \tau>0$.

Using this solution in the boundary condition (16), we obtain the algebraic system

$$
\left[\begin{array}{cc}
-\alpha_{0}^{2} k_{\alpha} & -i \eta l \\
i \eta l & -\beta_{0}^{2} k_{\beta}
\end{array}\right]\left[\begin{array}{l}
R \\
S
\end{array}\right]=\left[\begin{array}{l}
0 \\
0
\end{array}\right]
$$

Hence, (15)-(16) has a nonzero solution of the form (17) if and only if $L(\tau, l)=0$, where

$$
L(\tau, l)=\alpha_{0}^{2} \beta_{0}^{2} k_{\alpha} k_{\beta}-\eta^{2} l^{2}
$$

is the Lopatinski determinant associated with the IBVP.

The IBVP is invariant under the rescaling $x \mapsto r x, y \mapsto r y, t \mapsto r t$, for all $r>0$, and the reflection $y \mapsto-y, v \mapsto-v$, so we may set $l=1$ without loss of generality. In that case, writing $k_{\alpha}=\gamma_{\alpha}$ and $k_{\beta}=\gamma_{\beta}$, we have

$$
L(\tau, 1)=\alpha_{0}^{2} \beta_{0}^{2} \gamma_{\alpha} \gamma_{\beta}-\eta^{2}, \quad \gamma_{\alpha}=\sqrt{1+\frac{\tau^{2}}{\alpha_{0}^{2}}}, \quad \gamma_{\beta}=\sqrt{1+\frac{\tau^{2}}{\beta_{0}^{2}}} .
$$

A necessary condition for the well-posedness of the IBVP forward in time is the Lopatinski condition [6]

$$
L(\tau, 1) \neq 0 \quad \text { for all } \tau \in \mathbb{C} \text { with } \Re \tau>0 .
$$


In other words, there are no Fourier-Laplace modes that oscillate in the tangential spatial direction, decay in the normal spatial direction, and grow in time. If such modes do exist, then the IBVP is catastrophically unstable, since rescaling them gives finite-energy modes that grow arbitrarily quickly in time.

We define

$$
a=\frac{\alpha_{0}^{2}}{|\eta|}, \quad b=\frac{\beta_{0}^{2}}{|\eta|}, \quad s^{2}=\frac{\tau^{2}}{|\eta|},
$$

where $0<a, b<\infty$. Then (19) is satisfied if and only if the quartic equation

$$
a b\left(a+s^{2}\right)\left(b+s^{2}\right)=1
$$

has no roots for $s$ with positive real part. Since

$$
s^{2}=\frac{1}{2}\left\{-(a+b) \pm \sqrt{\frac{4}{a b}+(a-b)^{2}}\right\}
$$

we get the following cases:

1. If $a b>1$, then $s^{2}<0$, and there are four imaginary roots for $\tau$, so (19) holds;

2. If $a b=1$, then $s^{2}<0$ or $s^{2}=0$, and there are two imaginary and one double-zero root for $\tau$, so (19) holds;

3. If $0<a b<1$, then $s^{2}<0$ or $s^{2}>0$, and there are two imaginary and two real roots for $\tau$, one of which is positive, so (19) fails.

Thus, neglecting the marginal case $a b=1$, we see that (15)-(16) satisfies the Lopatinski condition (19) if $a b>1$, or $\alpha_{0} \beta_{0}>|\eta|$. We assume from now on that this condition is satisfied.

\section{Linearized surface waves}

Next, we consider surface wave solutions of the linearized model IBVP, which correspond to Fourier-Laplace modes with a purely imaginary frequency $\tau$ that oscillate in time. The discussion in Section 2 shows that these modes exist for all parameter values.

We distinguish between two types of surface waves: (i) radiative, or leaky, waves that oscillate but do not decay in the normal spatial direction; (ii) genuine, or finite-energy, waves that decay exponentially in the normal spatial 
direction. Radiative surface waves are coupled with bulk waves in the interior of the half-space, whereas genuine surface waves are localized near the boundary.

To analyze the surface waves in more detail, it is convenient to write

$$
\tau=-i \lambda, \quad r^{2}=\frac{\lambda^{2}}{|\eta|}=-s^{2},
$$

where $\lambda \in \mathbb{R}$. Since we have normalized the tangential wavenumber $l$ to one, $\lambda$ is the speed of the surface wave along the boundary. The normal spatial decay constants in (18) are then given by

$$
\gamma_{\alpha}=\sqrt{1-\frac{r^{2}}{a}}, \quad \gamma_{\beta}=\sqrt{1-\frac{r^{2}}{b}} .
$$

For the negative square root in (21), we get

$$
r^{2}=\frac{1}{2}\left\{(a+b)+\sqrt{\frac{4}{a b}+(a-b)^{2}}\right\}>\frac{1}{2}\{(a+b)+|a-b|\}=\max (a, b) .
$$

Thus, $\gamma_{\alpha}$ and $\gamma_{\beta}$ are both purely imaginary, and the surface waves are radiative. In this case, $|\lambda|>\max \left(\alpha_{0}, \beta_{0}\right)$, meaning that the surface waves are faster than the bulk waves.

On the other hand, for the positive square root in (21), we get

$$
r^{2}=\frac{1}{2}\left\{(a+b)-\sqrt{\frac{4}{a b}+(a-b)^{2}}\right\}<\frac{1}{2}\{(a+b)-|a-b|\}=\min (a, b) .
$$

Thus, $\gamma_{\alpha}$ and $\gamma_{\beta}$ are both real and positive, and the surface waves are genuine, with both $u$ and $v$ decaying exponentially in the normal spatial direction. In this case, $|\lambda|<\min \left(\alpha_{0}, \beta_{0}\right)$, meaning that the surface waves are slower than the bulk waves.

We remark that it is these genuine surface-wave modes that lead to instability as $a b$ decreases through 1 ; their wave-speeds coalesce at $\lambda=0$ and become complex. Hunter and Thoo [20] carry out a bifurcation analysis of a similar problem in MHD.

The aim of this paper is to study the effect of weak nonlinearity on genuine variational surface waves. These problems typically lead to nonlocal asymptotic equations because the surface-wave speed is slower than the bulk-wave speeds, so that what happens at one point of the boundary can influence what happens elsewhere on the boundary through the half-space. 
The qualitative behavior of radiative surface waves, which are faster than the bulk waves, is different from that of the genuine surface waves, and one typically obtains local asymptotic equations. Weakly nonlinear radiative surface waves in this problem could be analyzed in a similar way to the radiative surface waves on a compressible vortex sheet studied in [4].

\section{Weakly nonlinear surface waves}

Weakly nonlinear, genuine surface wave solutions of the model IBVP (9)(10) may be derived by standard multiple-scale methods. The dominant nonlinear effects on surface waves are quadratic; for waves whose amplitude is of the order $\epsilon \ll 1$, they become significant on time-scales of the order $\epsilon^{-1}$ in a reference frame moving with the linearized wave speed.

In this section, we outline the multiple-scale expansion. Some details of the (lengthy) algebraic computations are given in the Appendix. In the next section, we outline an alternative derivation based on an expansion of the surface-wave Hamiltonian.

We introduce "fast" space variables $\theta=y-\lambda t$ and $x$, tangent and normal to the boundary, respectively, and a "slow" time variable $\tau=\epsilon t$. (We do not use $\tau$ to denote a complex frequency in this section, so this notation should cause no confusion.) We assume that the wave speeds $\alpha(u)$ and $\beta(v)$ in (15) have the expansions

$$
\alpha^{2}(u)=\alpha_{0}^{2}+2 \alpha_{0} \alpha_{1} u+O\left(u^{2}\right), \quad \beta^{2}(u)=\beta_{0}^{2}+2 \beta_{0} \beta_{1} v+O\left(v^{2}\right)
$$

as $u, v \rightarrow 0$, where $\alpha_{0}=\alpha(0), \beta_{0}=\beta(0), \alpha_{1}=\alpha^{\prime}(0), \beta_{1}=\beta^{\prime}(0)$, and $\alpha_{0}, \beta_{0}>0$. We then seek an asymptotic expansion as $\epsilon \rightarrow 0$ for a solution $(u, v)$ of $(15)-(16)$ of the form

$$
\begin{aligned}
& u(x, y, t ; \epsilon)=\epsilon u_{1}(x, y-\lambda t, \epsilon t)+\epsilon^{2} u_{2}(x, y-\lambda t, \epsilon t)+O\left(\epsilon^{3}\right), \\
& v(x, y, t ; \epsilon)=\epsilon v_{1}(x, y-\lambda t, \epsilon t)+\epsilon^{2} v_{2}(x, y-\lambda t, \epsilon t)+O\left(\epsilon^{3}\right),
\end{aligned}
$$

where $\lambda$ is the linearized surface wave speed. The leading-order approximations $u_{1}(x, \theta, \tau), v_{1}(x, \theta, \tau)$ are determined from the requirement that this expansion is formally valid for times $t=O\left(\epsilon^{-1}\right)$.

At the order $\epsilon$, we find that $\left(u_{1}, v_{1}\right)$ satisfies the linearized equations. The solution for $\left(u_{1}, v_{1}\right)$ is a linear superposition of the Fourier-Laplace modes described in the previous section and is given by

$$
\left[\begin{array}{l}
u_{1} \\
v_{1}
\end{array}\right](x, \theta, \tau)=\int_{\mathbb{R}} \hat{a}(k, \tau)\left[\begin{array}{l}
R(k) e^{-\gamma_{\alpha}|k| x} \\
S(k) e^{-\gamma_{\beta}|k| x}
\end{array}\right] e^{i k \theta} d k,
$$


where $\hat{a}(k, \tau)$ is an arbitrary complex-valued amplitude-function of the tangential wavenumber $k$ and the slow time $\tau$, with $\hat{a}(-k, \tau)=\hat{a}^{*}(k, \tau)$.

The decay constants

$$
\gamma_{\alpha}=\sqrt{1-\frac{\lambda^{2}}{\alpha_{0}^{2}}}, \quad \gamma_{\beta}=\sqrt{1-\frac{\lambda^{2}}{\beta_{0}^{2}}}
$$

in (24) satisfy equation (18),

$$
\alpha_{0}^{2} \beta_{0}^{2} \gamma_{\alpha} \gamma_{\beta}=\eta^{2},
$$

and the wave speed $\lambda$ is given by

$$
\lambda^{2}=\frac{1}{2}\left\{\alpha_{0}^{2}+\beta_{0}^{2}-\sqrt{\left(\alpha_{0}^{2}-\beta_{0}^{2}\right)^{2}+\frac{4 \eta^{4}}{\alpha_{0}^{2} \beta_{0}^{2}}}\right\} .
$$

Here, we assume the stability condition $\alpha_{0} \beta_{0}>|\eta|$, and we choose the sign of the square root that corresponds to genuine surface waves. The coefficients $R$ and $S$ in (24) give the relative amplitudes of $u$ and $v$ in the surface wave, and are determined up to a scalar factor. A convenient choice is

$$
R=\eta, \quad S=i \alpha_{0}^{2} \gamma_{\alpha} \operatorname{sgn}(k) .
$$

At the order $\epsilon^{2}$, we find that $\left(u_{2}, v_{2}\right)$ satisfies a nonhomogeneous linearized system, where the nonhomogeneous term depends on $\left(u_{1}, v_{1}\right)$. A solution for $\left(u_{2}, v_{2}\right)$ exists only if the nonhomogeneous term satisfies an appropriate solvability condition, which yields an evolution equation for the amplitude-function $\hat{a}(k, \tau)$. After some algebra, we get (11)-(12) stated in the introduction, with

$$
\begin{aligned}
& E_{0}=\lambda\left[\frac{1}{\gamma_{\alpha}^{2}}+\frac{1}{\gamma_{\beta}^{2}}-2\right]^{-1}, \\
& A_{0}=\frac{\eta \alpha_{1}}{\alpha_{0}}, \quad B_{0}=\frac{\alpha_{0}^{2} \gamma_{\alpha} \beta_{1}}{\beta_{0}}, \quad C_{0}=\frac{A_{0}}{\gamma_{\alpha}^{2}}, \quad D_{0}=\frac{B_{0}}{\gamma_{\beta}^{2}} .
\end{aligned}
$$

In summary, the weakly nonlinear, genuine surface wave solution of (9)-(10) has the asymptotic expansion (23)-(24), where the spectral waveamplitude $\hat{a}(k, \tau)$ satisfies (11). Assuming that we have "prepared" initial data of the same form as (24), corresponding to a unidirectional surface wave, we supplement (11) with an initial condition $\hat{a}(k, 0)=\hat{a}_{0}(k)$. 


\section{Hamiltonian equations}

In this section, we derive equation (11) for a weakly nonlinear surface wave solution of (9)-(10) by expanding its Hamiltonian up to cubic terms in the wave amplitude and evaluating the cubic terms on the linearized surface wave solution. This procedure is somewhat heuristic, but it involves less algebra than the multiple-scale approach and, as we will verify, it leads to the same result. It also provides an independent check on the multiple-scale analysis. See [30] for further explanation of the Hamiltonian formalism we use here.

\subsection{Expansion of the Hamiltonian}

The variational principle for the model IBVP (9)-(10) is (7)-(8). We denote the canonically conjugate momenta to $u$ and $v$ by $p=u_{t}$ and $q=v_{t}$, respectively. The corresponding Hamiltonian functional $\mathcal{H}$ is given by

$$
\mathcal{H}(u, v, p, q)=\mathcal{A}(u, p)+\mathcal{B}(v, q)+\mathcal{E}(u, v)
$$

where

$$
\begin{aligned}
& \mathcal{A}(u, p)=\frac{1}{2} \int_{\mathbb{R}_{+}^{2}}\left[p^{2}+\alpha^{2}(u)\left(u_{x}^{2}+u_{y}^{2}\right)\right] d x d y \\
& \mathcal{B}(v, q)=\frac{1}{2} \int_{\mathbb{R}_{+}^{2}}\left[q^{2}+\beta^{2}(v)\left(v_{x}^{2}+v_{y}^{2}\right)\right] d x d y \\
& \mathcal{E}(u, v)=\int_{\mathbb{R}_{+}^{2}} \eta\left[u_{y} v_{x}-u_{x} v_{y}\right] d x d y .
\end{aligned}
$$

Using the Taylor expansion (22) of the wave speeds in $\mathcal{A}$ and $\mathcal{B}$, we get that

$$
\mathcal{A}=\mathcal{A}_{2}+\mathcal{A}_{3}+O\left(u^{4}\right), \quad \mathcal{B}=\mathcal{B}_{2}+\mathcal{B}_{3}+O\left(v^{4}\right)
$$

where

$$
\begin{aligned}
& \mathcal{A}_{2}=\frac{1}{2} \int_{\mathbb{R}_{+}^{2}}\left[p^{2}+\alpha_{0}^{2}\left(u_{x}^{2}+u_{y}^{2}\right)\right] d x d y, \quad \mathcal{A}_{3}=\alpha_{0} \alpha_{1} \int_{\mathbb{R}_{+}^{2}} u\left[u_{x}^{2}+u_{y}^{2}\right] d x d y \\
& \mathcal{B}_{2}=\frac{1}{2} \int_{\mathbb{R}_{+}^{2}}\left[q^{2}+\beta_{0}^{2}\left(v_{x}^{2}+v_{y}^{2}\right)\right] d x d y, \quad \mathcal{B}_{3}=\beta_{0} \beta_{1} \int_{\mathbb{R}_{+}^{2}} v\left[v_{x}^{2}+v_{y}^{2}\right] d x d y
\end{aligned}
$$

Thus, the expansion of the Hamiltonian as $u, v \rightarrow 0$ is

$$
\mathcal{H}=\mathcal{H}_{2}+\mathcal{H}_{3}+O\left(u^{4}+v^{4}\right)
$$


where the quadratic and cubic terms $\mathcal{H}_{2}$ and $\mathcal{H}_{3}$, respectively, are given by

$$
\mathcal{H}_{2}=\mathcal{A}_{2}+\mathcal{B}_{2}+\mathcal{E}, \quad \mathcal{H}_{3}=\mathcal{A}_{3}+\mathcal{B}_{3} .
$$

To apply the Hamiltonian formalism, we separate the positive and negative frequency components of the wave amplitude. The linearized frequency of the surface wave is given by

$$
\omega(k)=\lambda k
$$

where the wave speed $\lambda$ satisfies (27). For definiteness, we consider rightmoving waves with $\lambda>0$. In that case, the positive frequency components are the ones with positive wavenumbers, and the spatial Fourier coefficients

$$
\left\{\tilde{a}(k, t), \tilde{a}^{*}(k, t): k>0\right\}
$$

are complex-canonical conjugate variables for the wave, provided they are scaled appropriately.

The expression for $(u, v, p, q)$ in a unidirectional, linear surface wave solution has the form

$$
\begin{aligned}
& u=C_{0} \int_{0}^{\infty}\left[R \tilde{a}(k, t) e^{i k y-\gamma_{\alpha} k x}+R^{*} \tilde{a}^{*}(k, t) e^{-i k y-\gamma_{\alpha} k x}\right] d k, \\
& v=C_{0} \int_{0}^{\infty}\left[S \tilde{a}(k, t) e^{i k y-\gamma_{\beta} k x}+S^{*} \tilde{a}^{*}(k, t) e^{-i k y-\gamma_{\beta} k x}\right] d k, \\
& p=C_{0} \int_{0}^{\infty} i \omega(k)\left[R \tilde{a}(k, t) e^{i k y-\gamma_{\alpha} k x}-R^{*} \tilde{a}^{*}(k, t) e^{-i k y-\gamma_{\alpha} k x}\right] d k, \\
& q=C_{0} \int_{0}^{\infty} i \omega(k)\left[S \tilde{a}(k, t) e^{i k y-\gamma_{\beta} k x}-S^{*} \tilde{a}^{*}(k, t) e^{-i k y-\gamma_{\beta} k x}\right] d k,
\end{aligned}
$$

where $\gamma_{\alpha}, \gamma_{\beta}$ are defined in (25). Moreover, from (28), we have for $k>0$ that

$$
R=\eta, \quad S=i \alpha_{0}^{2} \gamma_{\alpha}
$$

We choose the positive scaling constant $C_{0}$ in (35) below, after we compute $\mathcal{H}_{2}$.

We use the linearized solution (32) in the Hamiltonian (31) and evaluate the resulting integrals with respect to $(x, y)$.

A straightforward computation, using Parseval's theorem, shows that the quadratic term in the Hamiltonian is given by

$$
\mathcal{H}_{2}\left(\tilde{a}, \tilde{a}^{*}\right)=\sigma_{0} C_{0}^{2} \int_{0}^{\infty} \lambda k \tilde{a}^{*}(k, t) \tilde{a}(k, t) d k,
$$


where

$$
\sigma_{0}=\frac{\rho_{0}}{E_{0}}, \quad \rho_{0}=2 \pi \alpha_{0}^{2} \gamma_{\alpha}|R|^{2}=2 \pi \beta_{0}^{2} \gamma_{\beta}|S|^{2}
$$

and $E_{0}$ is defined in (29). We note that $\sigma_{0}>0$ for $\lambda>0$. When expressed in terms of complex canonical variables, the quadratic part of the Hamiltonian should have the form [30]

$$
\mathcal{H}_{2}\left(\tilde{a}, \tilde{a}^{*}\right)=\int_{0}^{\infty} \omega(k) \tilde{a}^{*}(k, t) \tilde{a}(k, t) d k, \quad \omega(k)=\lambda k
$$

This is the case if we choose

$$
C_{0}=\frac{1}{\sqrt{\sigma_{0}}}
$$

A longer computation [5] shows that the cubic term in the Hamiltonian (31) is given by

$\mathcal{H}_{3}\left(\tilde{a}, \tilde{a}^{*}\right)=\rho_{0} C_{0}^{3} \int_{0}^{\infty} \int_{0}^{\infty} T(-l-m, l, m) \tilde{a}^{*}(l+m) \tilde{a}(l, t) \tilde{a}(m, t) d l d m+$ c.c. where $\rho_{0}$ is defined in (34) and the kernel $T$ may be written as

$$
\begin{aligned}
T\left(k_{1}, k_{2}, k_{3}\right) & =\left[\frac{R \alpha_{1}}{\alpha_{0}}+\frac{S \beta_{1}}{\beta_{0}}\right]\left[\frac{-k_{1} k_{2}+k_{2} k_{3}-k_{1} k_{3}}{-k_{1}+k_{2}+k_{3}}\right] \\
& -\left[\frac{R \alpha_{1}}{\alpha_{0} \gamma_{\alpha}^{2}}+\frac{S \beta_{1}}{\beta_{0} \gamma_{\beta}^{2}}\right]\left[\frac{k_{1} k_{2}+k_{2} k_{3}+k_{1} k_{3}}{-k_{1}+k_{2}+k_{3}}\right] .
\end{aligned}
$$

From (34) and (35), we have $\rho_{0} C_{0}^{2}=E_{0}$. Thus, neglecting quartic and higher-degree terms, our final expression for the expanded surface-wave Hamiltonian is

$$
\begin{aligned}
& \mathcal{H}\left(\tilde{a}, \tilde{a^{*}}\right)=\int_{0}^{\infty} \lambda k \tilde{a}^{*}(k, t) \tilde{a}(k, t) d k \\
& \quad+C_{0} E_{0} \int_{0}^{\infty} \int_{0}^{\infty} T(-l-m, l, m) \tilde{a}^{*}(l+m) \tilde{a}(l, t) \tilde{a}(m, t) d l d m \\
& \quad+C_{0} E_{0} \int_{0}^{\infty} \int_{0}^{\infty} T^{*}(-l-m, l, m) \tilde{a}(l+m, t) \tilde{a}^{*}(l, t) \tilde{a}^{*}(m, t) d l d m .
\end{aligned}
$$




\subsection{Hamilton's equation}

The complex canonical form of Hamilton's equation for $\left\{\tilde{a}(k, t): k \in \mathbb{R}_{+}\right\}$is

$$
i \tilde{a}_{t}(k, t)=\frac{\delta \mathcal{H}}{\delta \tilde{a}^{*}(k, t)},
$$

where $\delta \mathcal{H} / \delta \tilde{a}^{*}$ denotes the functional derivative of $\mathcal{H}\left(a, a^{*}\right)$ with respect to $\tilde{a}^{*}$, keeping $\tilde{a}$ fixed. Hamilton's equation for the Hamiltonian (37) is

$$
\begin{aligned}
i \tilde{a}_{t}(k, t)=\lambda k \tilde{a}(k, t) & +C_{0} E_{0} \int_{0}^{k} T(-k, k-l, l) \tilde{a}(k-l, t) \tilde{a}(l, t) d l \\
& +2 C_{0} E_{0} \int_{0}^{\infty} T^{*}(-k-l, k, l) \tilde{a}(k+l, t) \tilde{a}^{*}(l, t) d l .
\end{aligned}
$$

Following [3], we rewrite equation (39) for the positive wavenumber components $\left\{\tilde{a}(k, t): k \in \mathbb{R}_{+}\right\}$as a convolution-type equation for all of the wavenumber components $\{\tilde{a}(k, t): k \in \mathbb{R}\}$, where $\tilde{a}(-k, t)=\tilde{a}^{*}(k, t)$. The result is that

$$
i \tilde{a}_{t}(k, t)=\lambda k \tilde{a}(k)+C_{0} E_{0} \operatorname{sgn} k \int_{-\infty}^{\infty} \Lambda(-k, k-l, l) \tilde{a}(k-l, t) \tilde{a}(l, t) d l,
$$

where the kernel $\Lambda: \mathbb{R}^{3} \rightarrow \mathbb{C}$ is given in terms of the kernel $T: \mathbb{R}_{-} \times \mathbb{R}_{+}^{2} \rightarrow \mathbb{C}$ on $k_{1}+k_{2}+k_{3}$ as follows:

1. $\Lambda\left(k_{1}, k_{2}, k_{3}\right)=T\left(k_{1}, k_{2}, k_{3}\right)$ if $k_{2}, k_{3}>0$ and $k_{1}<0$;

2. $\Lambda\left(k_{1}, k_{2}, k_{3}\right)=T\left(k_{2}, k_{1}, k_{3}\right)$ if $k_{1}, k_{3}>0$ and $k_{2}<0$;

3. $\Lambda\left(k_{1}, k_{2}, k_{3}\right)=T\left(k_{3}, k_{1}, k_{2}\right)$ if $k_{1}, k_{2}>0$ and $k_{3}<0$;

4. $\Lambda\left(k_{1}, k_{2}, k_{3}\right)=T^{*}\left(-k_{1},-k_{2},-k_{3}\right)$ if $k_{2}, k_{3}<0$ and $k_{1}>0$;

5. $\Lambda\left(k_{1}, k_{2}, k_{3}\right)=T^{*}\left(-k_{2},-k_{1},-k_{3}\right)$ if $k_{1}, k_{3}<0$ and $k_{2}>0$;

6. $\Lambda\left(k_{1}, k_{2}, k_{3}\right)=T^{*}\left(-k_{3},-k_{1},-k_{2}\right)$ if $k_{1}, k_{2}<0$ and $k_{3}>0$.

By considering the different possible sign combinations of $k_{1}, k_{2}, k_{3}$, one can verify [5] that if $T\left(k_{1}, k_{2}, k_{3}\right)$ is given by (36), with $R$ and $S$ defined as in (33), then this expression for $\Lambda\left(k_{1}, k_{2}, k_{3}\right)$ agrees with (12) on $k_{1}+k_{2}+k_{3}=0$.

By comparing the multiple-scale solution (23)-(24) with the Hamiltonian solution (32), we see that the corresponding wave amplitudes are related by

$$
\tilde{a}(k, t)=\frac{\epsilon}{C_{0}} \hat{a}(k, \epsilon t) e^{-i \lambda k t} .
$$


The use of this expression for $\tilde{a}(k, t)$ in equation (40) gives equation (11) for $\hat{a}(k, \tau)$. The factor $e^{-i \lambda k t}$ in $\tilde{a}$ corresponds to a Galilean transformation which removes the linear term from (40). Thus, the Hamiltonian and multiple-scale approaches lead to identical results.

Finally, we remark that a similar procedure can be applied to the Lagrangian instead of the Hamiltonian. One expands the Lagrangian up to cubic terms in the field variables and then evaluates these terms on the linearized surface wave solution. This gives a variational principle whose Euler-Lagrange equation is the asymptotic equation.

\section{Spatial form of the asymptotic equation}

In this section, we show how to rewrite the spectral form of the asymptotic equation (11) in an equivalent spatial form, and we give a simplified, but representative, spatial equation.

We write (11) as

$$
\hat{a}_{\tau}(k, \tau)+i \operatorname{sgn}(k) E_{0} \hat{f}(k, \tau)=0
$$

where

$$
\hat{f}(k, \tau)=\int_{-\infty}^{\infty} \Lambda(-k, k-l, l) \hat{a}(k-l, \tau) \hat{a}(l, \tau) d l .
$$

Let

$$
a(\theta, \tau)=\int \hat{a}(k, \tau) e^{i k \theta} d \theta
$$

denote the spatial amplitude, with corresponding notation for $f$. Then, taking the inverse Fourier transform of (41), we get that $a_{\tau}=E_{0} H f$ where $H$ is the Hilbert transform with respect to $\theta$.

To express $f$ in terms of $a$, it is convenient to write the kernel $\Lambda$ in (12) as

$$
\begin{aligned}
\Lambda\left(k_{1}, k_{2}, k_{3}\right) & =\frac{1}{2}\left[\left(1-\frac{1}{\gamma_{\alpha}^{2}}\right) A_{0}-i \operatorname{sgn}\left(k_{1} k_{2} k_{3}\right)\left(1-\frac{1}{\gamma_{\beta}^{2}}\right) B_{0}\right] \Lambda_{+}\left(k_{1}, k_{2}, k_{3}\right) \\
& +\frac{1}{2}\left[\left(1+\frac{1}{\gamma_{\alpha}^{2}}\right) A_{0}-i \operatorname{sgn}\left(k_{1} k_{2} k_{3}\right)\left(1+\frac{1}{\gamma_{\beta}^{2}}\right) B_{0}\right] \Lambda_{-}\left(k_{1}, k_{2}, k_{3}\right),
\end{aligned}
$$

where

$$
\Lambda_{+}\left(k_{1}, k_{2}, k_{3}\right)=\frac{\left|k_{1} k_{2}\right|+\left|k_{2} k_{3}\right|+\left|k_{1} k_{3}\right|+k_{1} k_{2}+k_{2} k_{3}+k_{1} k_{3}}{\left|k_{1}\right|+\left|k_{2}\right|+\left|k_{3}\right|}
$$




$$
\Lambda_{-}\left(k_{1}, k_{2}, k_{3}\right)=\frac{\left|k_{1} k_{2}\right|+\left|k_{2} k_{3}\right|+\left|k_{1} k_{3}\right|-k_{1} k_{2}-k_{2} k_{3}-k_{1} k_{3}}{\left|k_{1}\right|+\left|k_{2}\right|+\left|k_{3}\right|} .
$$

The kernels $\Lambda_{ \pm}\left(k_{1}, k_{2}, k_{3}\right)$ may be written on $k_{1}+k_{2}+k_{3}=0$ in the equivalent forms

$$
\begin{aligned}
& \Lambda_{+}\left(k_{1}, k_{2}, k_{3}\right)=\frac{1}{2}\left[\frac{\left|k_{2} k_{3}\right|+k_{2} k_{3}}{\left|k_{1}\right|}+\frac{\left|k_{1} k_{3}\right|+k_{1} k_{3}}{\left|k_{2}\right|}+\frac{\left|k_{1} k_{2}\right|+k_{1} k_{2}}{\left|k_{3}\right|}\right], \\
& \Lambda_{-}\left(k_{1}, k_{2}, k_{3}\right)=\frac{1}{2}\left[\left|k_{1}\right|+\left|k_{2}\right|+\left|k_{3}\right|\right],
\end{aligned}
$$

as one can verify by considering the different sign combinations of $k_{1}, k_{2}, k_{3}$.

Using the expressions in (43), the fact that $|\partial|$ has symbol $|k|$, and the convolution theorem, we can read off the spatial terms in the equation that correspond to the spectral terms. For example, if

$$
\begin{aligned}
& \hat{f}_{+}(k)=\int \Lambda_{+}(-k, k-\xi, \xi) \hat{a}(k-\xi) \hat{a}(\xi) d \xi, \\
& \hat{f}_{-}(k)=\int \Lambda_{-}(-k, k-\xi, \xi) \hat{a}(k-\xi) \hat{a}(\xi) d \xi
\end{aligned}
$$

then

$$
\begin{aligned}
& f_{+}(\theta)=\frac{1}{2}|\partial|^{-1}\left\{(|\partial| a)^{2}-(\partial a)^{2}\right\}+|\partial|\left\{|\partial| a \cdot|\partial|^{-1} a\right\}+\partial\left\{\partial a \cdot|\partial|^{-1} a\right\}, \\
& f_{-}(\theta)=\frac{1}{2}|\partial|\left(a^{2}\right)+a|\partial| a .
\end{aligned}
$$

The terms in $\Lambda\left(k_{1}, k_{2}, k_{3}\right)$ with factors of $i \operatorname{sgn}\left(k_{1} k_{2} k_{3}\right)$ lead to additional Hilbert transforms on each factor and on the whole function.

The spatial form of the full asymptotic equation follows from the previous discussion, but it is somewhat lengthy, and we will not write it out explicitly here. Instead, we consider a simplification of the asymptotic equation in which $\Lambda$ only includes a $\Lambda_{-}$term. This corresponds to taking $\gamma_{\alpha}=\gamma_{\beta}=1$ in (42), which arises from the limit $\lambda \rightarrow 0$ in the original problem, and $B_{0}=0$, meaning that the only nonlinearity comes from the $u$-equation.

The derivation of the asymptotic equation does not apply when $\lambda=0$, since the linearized surface wave speeds coalesce at that point and the linear time-scale factor $E_{0}$ in (29) diverges as $\lambda \rightarrow 0$. Nevertheless, if $\lambda$ is small, it is reasonable to approximate the coefficients of the kernel $\Lambda$, which describe the effects of nonlinearity, by their values at $\lambda=0$. 
We then get the spectral equation

$$
\hat{a}_{\tau}(k, \tau)+\frac{1}{2} i \operatorname{sgn}(k) A_{0} E_{0} \int_{-\infty}^{\infty}(|k|+|k-l|+|l|) \hat{a}(k-l, \tau) \hat{a}(l, \tau) d l=0 .
$$

The corresponding spatial equation for $a(\theta, \tau)$ is

$$
a_{\tau}+A_{0} E_{0}\left\{\left(\frac{1}{2} a^{2}\right)_{\theta}-H[a|\partial| a]\right\}=0
$$

\section{A simplified asymptotic equation}

In this section, we consider the simplified asymptotic equation (44). We write $(x, t)$ instead of $(\theta, \tau)$, so $x$ is now a tangential spatial variable, and rescale $a$ to remove the inessential constant $A_{0} E_{0}$.

After this change in notation, we get the following equation for $a(x, t)$

$$
a_{t}+\left(\frac{1}{2} a^{2}\right)_{x}=H[a|\partial| a]
$$

where $H$ is the Hilbert transform with respect to $x$ and $|\partial|=H \partial_{x}$. We can also write (45) as

$$
a_{t}+\left(a^{2}\right)_{x}=[a, H]|\partial| a,
$$

where $[a, H]$ denotes the commutator of multiplication by $a$ and $H$. The right-hand side of (46) satisfies the estimate

$$
\|[a, H]|\partial| a\|_{L^{2}} \leq C\|a\|_{\dot{H}^{1 / 2}}^{2},
$$

so it is a lower-order term for smooth solutions.

The Hamiltonian form of (45) is

$$
a_{t}=H\left[\frac{\delta \mathcal{H}}{\delta a}\right], \quad \mathcal{H}(a)=\frac{1}{2} \int a^{2}|\partial| a d x
$$

The Hilbert transform $H$ is the spatial Hamiltonian operator corresponding to the spectral Hamiltonian operator of multiplication by $-i$ on positive wavenumbers. We can consider either free-space solutions of (45), with $x \in \mathbb{R}$, or spatially periodic solutions, with $x \in \mathbb{T}$, and interpret integrals over $x$ as appropriate. 
The Hamiltonian $\mathcal{H}$ is conserved by smooth solutions of (45), but this is not particularly useful for analytical purposes since $\mathcal{H}$ is cubic and indefinite. An additional positive conserved quantity, associated with the invariance of (45) under spatial translations, is the momentum

$$
\mathcal{P}(a)=\frac{1}{2} \int a|\partial| a d x .
$$

The mean $\int a d x$ is also conserved, but we do not know of any other conserved quantities for (45).

The momentum $\mathcal{P}$ provides an a priori $H^{1 / 2}$-estimate. It also plays the role of an entropy for dissipative weak solutions of (45). To show this, we consider the viscous equation

$$
a_{t}+\left(\frac{1}{2} a^{2}\right)_{x}=H[a|\partial| a]+\epsilon a_{x x} .
$$

One finds that smooth solutions satisfy

$$
\begin{aligned}
& (a|\partial| a)_{t}+\left(\frac{1}{2} a|\partial|\left(a^{2}\right)+a^{2}|\partial| a\right)_{x} \\
& \quad=\frac{1}{2} a_{x}|\partial|\left(a^{2}\right)+|\partial| a \cdot H[a|\partial| a]+\epsilon\left(a|\partial| a_{x}+a_{x}|\partial| a\right)_{x}-2 \epsilon a_{x}|\partial| a_{x} .
\end{aligned}
$$

Integrating this equation with respect to $x$, using the skew-adjointness of $H$, and assuming that the boundary terms vanish, we get that

$$
\frac{d}{d t} \int a|\partial| a d x=-2 \epsilon \int a_{x}|\partial| a_{x} d x \leq 0 .
$$

\subsection{Short-time existence of smooth solutions}

We consider the following initial-value problem (IVP) for $a(x, t)$

$$
\begin{aligned}
a_{t}+\left(\frac{1}{2} a^{2}\right)_{x} & =H[a|\partial| a], \\
a(x, 0) & =f(x) .
\end{aligned}
$$

where $f: \mathbb{T} \rightarrow \mathbb{R}$ is a $2 \pi$-periodic function with zero mean. We look for spatially periodic solutions with zero mean,

$$
a(x, t)=\sum_{k \in \mathbb{Z}} \hat{a}(k, t) e^{i k x},
$$


where $\hat{a}(0, t)=0$ and $\hat{a}(-k, t)=\hat{a}^{*}(k, t)$, and denote by $\dot{H}^{s}(\mathbb{T})$ the usual $L^{2}$-Sobolev space with norm

$$
\|a\|_{\dot{H}^{s}}=\left(\sum_{k \in \mathbb{Z}}|k|^{2 s}|\hat{a}(k)|^{2}\right)^{1 / 2}
$$

Theorem 7.1. Suppose that $f \in \dot{H}^{s}(\mathbb{T})$ where $s>3 / 2$. Then there is a unique local solution of (48) with

$$
a \in C\left(I ; \dot{H}^{s}\right) \cap C^{1}\left(I ; \dot{H}^{s-1}\right)
$$

defined on a time interval $I=(-T, T)$, where $T=C_{s} /\|f\|_{\dot{H}^{s}}$ for some constant $C_{s}>0$.

Proof. The proof is by a standard Galerkin method [28]. We omit the details and just give the required a priori energy estimate following [17]. The same estimate and proof applies to equations with the more general kernels (12) or $(71)$.

The spectral form of (48) is

$$
\hat{a}_{t}(k, t)+\frac{1}{2} i \operatorname{sgn} k \sum_{\xi \in \mathbb{Z}}(|k|+|k-\xi|+|\xi|) \hat{a}(k-\xi, t) \hat{a}(\xi, t)=0 .
$$

It follows that

$$
\begin{aligned}
& \frac{d}{d t} \sum_{k \in \mathbb{Z}}|k|^{2 s+1}|\hat{a}(k, t)|^{2} \\
& \quad+i \sum_{k, \xi \in \mathbb{Z}} k|k|^{2 s}(|k|+|k-\xi|+|\xi|) \hat{a}(-k, t) \hat{a}(k-\xi, t) \hat{a}(\xi, t)=0 .
\end{aligned}
$$

Using the symmetry of the kernel, we can write this equation as $\frac{d}{d t} \sum_{k \in \mathbb{Z}}|k|^{2 s+1}|\hat{a}(k, t)|^{2}+\frac{1}{3} i \sum_{k, \xi \in \mathbb{Z}} \Lambda_{s}(-k, k-\xi, \xi) \hat{a}(-k, t) \hat{a}(k-\xi, t) \hat{a}(\xi, t)=0$

where

$$
\Lambda_{s}\left(k_{1}, k_{2}, k_{3}\right)=\left(k_{1}\left|k_{1}\right|^{2 s}+k_{2}\left|k_{2}\right|^{2 s}+k_{3}\left|k_{3}\right|^{2 s}\right)\left(\left|k_{1}\right|+\left|k_{2}\right|+\left|k_{3}\right|\right) .
$$


The following inequalities [17] hold on $k_{1}+k_{2}+k_{3}$ :

$$
\begin{aligned}
& \left|k_{1}\right|+\left|k_{2}\right|+\left|k_{3}\right| \leq \frac{2 \sqrt{2}\left|k_{1} k_{2} k_{3}\right|^{1 / 2}}{\min \left(\left|k_{1}\right|^{1 / 2},\left|k_{2}\right|^{1 / 2},\left|k_{3}\right|^{1 / 2}\right)}, \\
& \frac{\left.\left|k_{1}\right| k_{1}\right|^{2 s}+k_{2}\left|k_{2}\right|^{2 s}+k_{3}\left|k_{3}\right|^{2 s} \mid}{\min \left(\left|k_{1}\right|^{1 / 2},\left|k_{2}\right|^{1 / 2},\left|k_{3}\right|^{1 / 2}\right)} \\
& \quad \leq C_{s}\left(\left|k_{1}\right|^{s}\left|k_{2}\right|^{s}\left|k_{3}\right|^{1 / 2}+\left|k_{2}\right|^{s}\left|k_{3}\right|^{s}\left|k_{1}\right|^{1 / 2}+\left|k_{3}\right|^{s}\left|k_{1}\right|^{s}\left|k_{2}\right|^{1 / 2}\right),
\end{aligned}
$$

where $C_{s}$ denotes a generic constant depending on $s>0$. Using these inequalities, we get that

$$
\begin{aligned}
& \left|\Lambda_{s}\left(k_{1}, k_{2}, k_{3}\right)\right| \\
& \leq C_{s}\left(\left|k_{1}\right|^{s+1 / 2}\left|k_{2}\right|^{s+1 / 2}\left|k_{3}\right|+\left|k_{2}\right|^{s+1 / 2}\left|k_{3}\right|^{s+1 / 2}\left|k_{1}\right|+\left|k_{3}\right|^{s+1 / 2}\left|k_{1}\right|^{s+1 / 2}\left|k_{2}\right|\right)
\end{aligned}
$$

on $k_{1}+k_{2}+k_{3}=0$. By the Cauchy-Schwartz and Young inequalities, we can then estimate

$$
\begin{aligned}
\mid \sum_{k, \xi \in \mathbb{Z}} \Lambda_{s}( & -k, k-\xi, \xi) \hat{a}(-k, t) \hat{a}(k-\xi, t) \hat{a}(\xi, t) \mid \\
& \leq C_{s}\left\langle|k|^{s+1 / 2}|\hat{a}|,|k|^{s+1 / 2}|\hat{a}| *|k||\hat{a}|\right\rangle \\
& \leq C_{s}\left\||k|^{s+1 / 2} \hat{a}\right\|_{\ell^{2}} \cdot\left\|\left.|| k\right|^{s+1 / 2}|\hat{a}| *|k||\hat{a}|\right\|_{\ell^{2}} \\
& \leq C_{s}\left\||k|^{s+1 / 2} \hat{a}\right\|_{\ell^{2}}^{2}\||k| \hat{a}\|_{\ell^{1}},
\end{aligned}
$$

where, as usual,

$$
\langle|\hat{a}|,|\hat{b}|\rangle=\sum_{k \in \mathbb{Z}}|\hat{a}(k)||\hat{b}(k)|, \quad\|\hat{a}\|_{\ell^{p}}=\left(\sum_{k \in \mathbb{Z}}|\hat{a}(k)|^{p}\right)^{1 / p} .
$$

Since $\||k| \hat{a}\|_{\ell^{1}} \leq C_{r}\left\||k|^{r} \hat{a}\right\|_{\ell^{2}}$ for $r>3 / 2$, we conclude that

$$
\frac{d}{d t} \sum_{k \in \mathbb{Z}}|k|^{2 s+1}|\hat{a}(k, t)|^{2} \leq C_{s}\left(\sum_{k \in \mathbb{Z}}|k|^{2 s+1}|\hat{a}(k, t)|^{2}\right)^{3 / 2}
$$

if $s+1 / 2>3 / 2$, which gives an a priori $\dot{H}^{r}$-estimate for $a$ if $r>3 / 2$. 


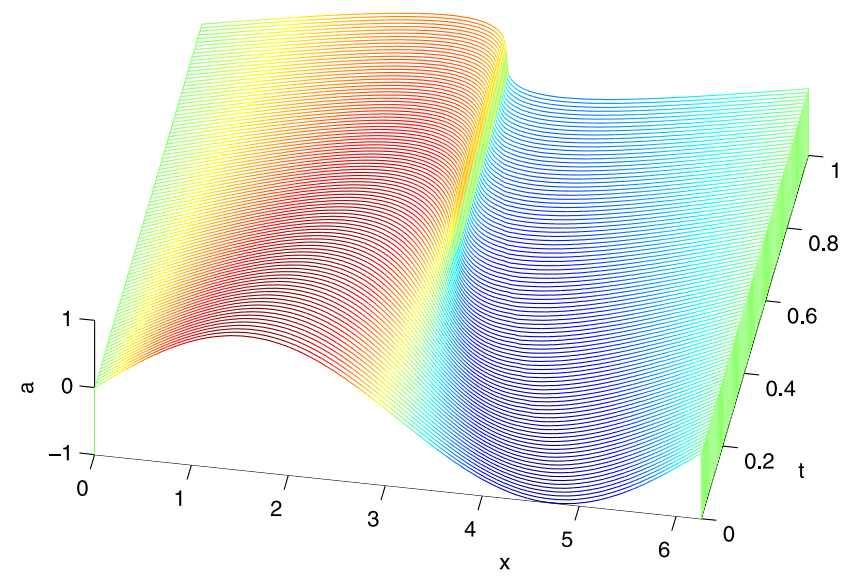

Figure 1: Numerical solution of (48) for $0 \leq t \leq 1$ with initial data $f(x)=$ $\sin x$.

The number of $L^{2}$-derivatives, $s>3 / 2$, that are required for local existence for $(45)$ is the same as the number required for local existence for the inviscid Burgers equation.

\subsection{Numerical solutions}

In this section, we show some numerical solutions of (48), which indicate that the spatial derivatives of smooth solutions blow up in finite time and that smooth solutions can be continued by weak dissipative solutions. The weak solutions appear to remain continuous after singularities form, with cusps rather than shocks, and they become continuous even if the initial data contains jump discontinuities.

We use a standard pseudo-spectral method with spectral viscosity and a fourth-order Runge-Kutta method in time. These numerical solutions are dissipative in nature; we do not address here the question whether or not (48) also has conservative weak solutions, as is the case for the HS equation that describes the corresponding bulk waves.

Figure 1 shows a surface plot of the solution with sinusoidal initial data for times $0 \leq t \leq 1$. The solution steepens in a similar way to solutions of the inviscid Burgers equation, and its derivative $a_{x}$ blows up at $t \approx 0.55$. This singularity formation time is a little longer than the time $t=0.5$ one would get by neglecting the lower-order term on the right-hand side of (46).

Figure 2 plots the momentum $\mathcal{P}$ in (47) of this solution as a function of time and shows the numerically computed spectrum at $t=1$. The momen- 

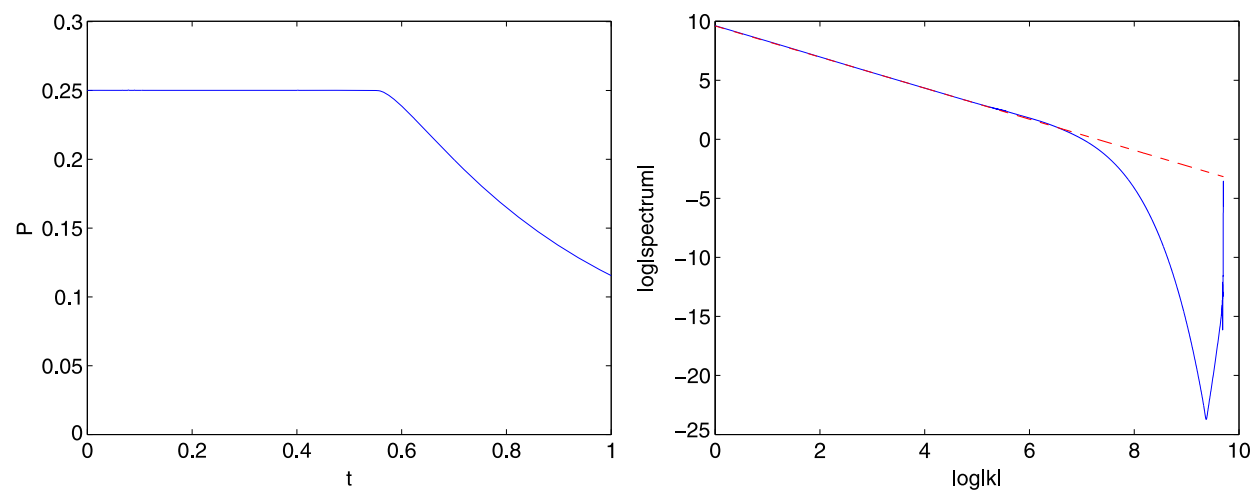

Figure 2: Left: The momentum $\mathcal{P}$ defined in (47) as a function of time for the solution shown in Figure 1. Right: The numerical spectrum $\log |\hat{a}(k, t)|$ at $t=1.0$ as a function of $\log |k|$. The dashed line is a best linear fit to the spectrum for $8 \leq|k| \leq 128$, and is $\log |\hat{a}(k, t)| \approx-1.315 \cdot \log |k|+9.591$. The numerical solution is computed for wavenumbers $|k| \leq 2^{14}$, after dealiasing. Spectral viscosity is switched on starting at $|k|=256$.

tum is constant until the singularity forms; after that it decreases. In contrast to the inviscid Burgers equation, the solution appears to remain continuous even after its derivative blows up. The power-law numerical spectrum of the solution at $t=1$, together with the following analytical solution, suggests that solutions have an $x^{\alpha}$-singularity with $\alpha \approx 1 / 3$.

If $\alpha$ is not an odd integer, then

$$
H\left[|x|^{\alpha}\right]=-C_{\alpha} \operatorname{sgn}(x)|x|^{\alpha}, \quad C_{\alpha} H\left[\operatorname{sgn}(x)|x|^{\alpha}\right]=|x|^{\alpha}, \quad C_{\alpha}=\tan \left(\frac{\pi \alpha}{2}\right)
$$

in a distributional sense. Using this formula, we find that

$$
a(x)=a_{0} \operatorname{sgn}(x)|x|^{\alpha}
$$

is a distributional solution of the steady equation

$$
\left(\frac{1}{2} a^{2}\right)_{x}=H[a|\partial| a]
$$

if $\alpha$ satisfies $C_{2 \alpha-1}+C_{\alpha}=0$. The solutions of this equation are

$$
\alpha=2 n \pm \frac{1}{3}, \quad n \in \mathbb{Z} .
$$




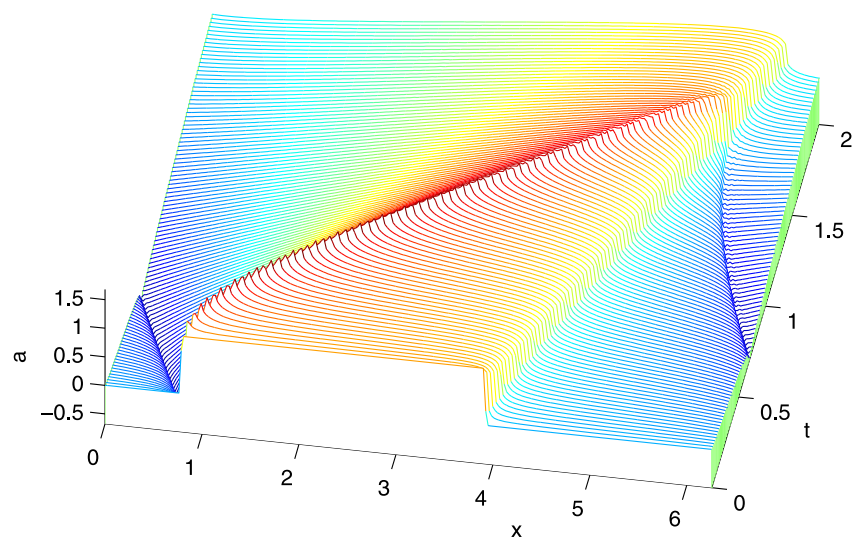

Figure 3: Numerical solution of (48) with square-wave initial data (50).

The smallest positive solution is $\alpha=1 / 3$. The corresponding spectral power law $|\hat{a}(k)|=\hat{a}_{0}|k|^{-4 / 3}$ is in approximate agreement with the numerical spectrum shown in Figure 2; there appears to be, however, a slight discrepancy, and our numerically computed spectrum has an exponent $\alpha$ that is below 1.32 .

This numerical solution of (45) is qualitatively similar to the numerical solution of the HIZ equation (5) with sinusoidal initial data, where a cusp singularity like (49) with $\alpha=2 / 3$ appears to form [18].

In Figure 3, we show a numerical solution of (48) with discontinuous square-wave initial data

$$
f(x)= \begin{cases}1 & \text { for } \pi / 4<x<5 \pi / 4 \\ 0 & \text { for } 0<x<\pi / 4 \text { or } 5 \pi / 4<x<2 \pi\end{cases}
$$

The global structure of the solution is similar to that of the solution for an inviscid Burgers equation, but it has a continuous cusp singularity instead of a shock and additional cusp singularities at each edge of the "expansion fan." In a periodic domain, these singularities hit each other and coalesce into one singularity.

\section{Orientation waves in a director field}

We conclude this paper with a description of a weakly nonlinear surface wave solution of the director-field equation. The derivation parallels the one for the model equation, but the algebra is more involved and we will 
only summarize the results. Additional details of the computations are given in $[5]$.

We consider a half-space IBVP in $\mathbb{R}_{+}^{3}=\{(x, y, z): x>0\}$ for the system of nonlinear hyperbolic partial differential equations for a massive director field that is obtained from the variational principle (2)-(3). The Euler-Lagrange equation in $x>0$ is

$$
\begin{aligned}
\mathbf{n}_{t t}=\alpha & \nabla(\operatorname{div} \mathbf{n})-\beta[\operatorname{curl}(A \mathbf{n})+A \operatorname{curl} \mathbf{n}] \\
& +\gamma[\mathbf{B} \times \operatorname{curl} \mathbf{n}-\operatorname{curl}(\mathbf{B} \times \mathbf{n})]+\lambda \mathbf{n}
\end{aligned}
$$

where $A=\mathbf{n} \cdot \operatorname{curl} \mathbf{n}$ and $\mathbf{B}=\mathbf{n} \times \operatorname{curl} \mathbf{n}$; the natural boundary condition on $x=0$ is

$$
(\alpha-\eta)(\operatorname{div} \mathbf{n}) \nu+\beta(A \mathbf{n} \times \nu)+\eta\left(\nu \cdot(\nabla \mathbf{n})^{T}\right)=\mu \mathbf{n},
$$

where $\nu=(-1,0,0)$ is the outward unit normal on the boundary $\partial \mathbb{R}_{+}^{3}$. The scalar fields $\lambda$ and $\mu$ in (51) and (52) are Lagrange multipliers that enforce the constraint $\mathbf{n} \cdot \mathbf{n}=1$ in the interior and on the boundary. They are given explicitly by

$$
\begin{aligned}
& \lambda=-\left|\mathbf{n}_{t}\right|^{2}+\alpha\left[|\nabla \mathbf{n}|^{2}-|\operatorname{curl} \mathbf{n}|^{2}\right]+\left[\beta A^{2}+\gamma|\mathbf{B}|^{2}\right]+(\alpha-\gamma) \operatorname{div} \mathbf{B} \\
& \mu=(\alpha-\eta)(\operatorname{div} \mathbf{n})(\nu \cdot \mathbf{n})
\end{aligned}
$$

This system is more complicated than the model system. Due to the anisotropic nature of the equations, the behavior of solutions depends on the direction of wave propagation and on the direction of the normal to the boundary. Nevertheless, we get asymptotic equations for weakly nonlinear surface waves that are qualitatively similar to the ones arising from the model system.

For definiteness, we consider only the case of surface waves that are small perturbations of a constant director field $\mathbf{n}_{0}=(0,0,1)$ that is tangent to the boundary.

\subsection{The Lopatinski condition}

We expand the solution of (51)-(52) as $\epsilon \rightarrow 0$ as

$$
\mathbf{n}(\mathbf{x}, t)=\mathbf{n}_{0}+\epsilon \mathbf{n}^{\prime}(\mathbf{x}, t)+O\left(\epsilon^{2}\right), \quad \mathbf{n}_{0}=(0,0,1) .
$$

Since $\mathbf{n}$ is a unit vector, $\mathbf{n}^{\prime}$ is orthogonal to $\mathbf{n}_{0}$, and we write it as

$$
\mathbf{n}^{\prime}(\mathbf{x}, t)=(u(\mathbf{x}, t), v(\mathbf{x}, t), 0),
$$


where $\mathbf{x}=(x, y, z)$. The linearization of the Euler-Lagrange equation (51) in $x>0$ is

$$
\begin{aligned}
& u_{t t}=\alpha u_{x x}+\beta u_{y y}+\gamma u_{z z}+(\alpha-\beta) v_{x y}, \\
& v_{t t}=\beta v_{x x}+\alpha v_{y y}+\gamma v_{z z}+(\alpha-\beta) u_{x y},
\end{aligned}
$$

and the linearization of the boundary condition (52) on $x=0$ is

$$
\begin{aligned}
& -\alpha u_{x}+(\eta-\alpha) v_{y}=0 \\
& -\beta v_{x}+(\beta-\eta) u_{y}=0 .
\end{aligned}
$$

We look for Fourier-Laplace solutions of the form

$$
\left[\begin{array}{l}
u \\
v
\end{array}\right]=\left[\begin{array}{l}
\hat{u} e^{-k x} \\
\hat{v} e^{-k x}
\end{array}\right] e^{\tau t+i l y+i m z}
$$

where $l, m \in \mathbb{R}, \tau \in \mathbb{C}$ with $\Re(\tau)>0$, and $k \in \mathbb{C}$ with $\Re(k)>0$. Using (56) in (54), we find that (56) is a solution of the PDE if

$$
\left[\begin{array}{cc}
\tau^{2}-\alpha k^{2}+\beta l^{2}+\gamma m^{2} & (\alpha-\beta) i k l \\
(\alpha-\beta) i k l & \tau^{2}-\beta k^{2}+\alpha l^{2}+\gamma m^{2}
\end{array}\right]\left[\begin{array}{l}
\hat{u} \\
\hat{v}
\end{array}\right]=\left[\begin{array}{l}
0 \\
0
\end{array}\right]
$$

It follows that $(k, l, m, \tau)$ satisfies the linearized dispersion relation

$$
\left[\tau^{2}-\alpha k^{2}+\beta l^{2}+\gamma m^{2}\right]\left[\tau^{2}-\beta k^{2}+\alpha l^{2}+\gamma m^{2}\right]+(\alpha-\beta)^{2} k^{2} l^{2}=0,
$$

and

$$
\left[\begin{array}{l}
u \\
v
\end{array}\right]=R\left[\begin{array}{c}
i k_{\alpha} \\
l
\end{array}\right] e^{\tau t+i l y+i m z-\gamma_{\alpha} x}+S\left[\begin{array}{c}
-l \\
i k_{\beta}
\end{array}\right] e^{\tau t+i l y+i m z-\gamma_{\beta} x},
$$

where $R, S$ are arbitrary constants. The decay constants $\gamma_{\alpha}, \gamma_{\beta}$ in (57) are given by

$$
\gamma_{\alpha}=\sqrt{l^{2}+\frac{\tau^{2}+\gamma m^{2}}{\alpha}}, \quad \gamma_{\beta}=\sqrt{l^{2}+\frac{\tau^{2}+\gamma m^{2}}{\beta}},
$$

where we take the branch of the square root with positive real part.

Using (57) in the boundary condition (55), we obtain the algebraic equation

$$
\left[\begin{array}{cc}
\eta l^{2}+\gamma m^{2}+\tau^{2} & i \eta l \gamma_{\beta} \\
-i \eta l \gamma_{\alpha} & \eta l^{2}+\gamma m^{2}+\tau^{2}
\end{array}\right]\left[\begin{array}{c}
R \\
S
\end{array}\right]=\left[\begin{array}{l}
0 \\
0
\end{array}\right]
$$


This equation has a nontrivial solution if and only if $L(\tau, l, m)=0$, where $L$ is the Lopatinski determinant

$$
L(\tau, l, m)=\left(\eta l^{2}+\gamma m^{2}+\tau^{2}\right)^{2}-\eta^{2} l^{2} \gamma_{\alpha} \gamma_{\beta} .
$$

The Lopatinski condition for this problem is then

$$
L(\tau, l, m) \neq 0 \quad \text { for all } l, m \in \mathbb{R} \text { and } \tau \in \mathbb{C} \text { with } \Re(\tau)>0 .
$$

If the Lopatinski condition for a second-order, variational IBVP fails, then it fails for a real value of $\tau$ (see Theorem 3.4 in [27]). Thus, writing

$$
a=\frac{\alpha}{\eta}, \quad b=\frac{\beta}{\eta}, \quad c=\frac{\gamma}{\eta}, \quad X=\frac{\tau^{2}}{\eta},
$$

we see that the Lopatinski condition (60) is satisfied if and only if there are no $l, m \in \mathbb{R}$ with $l^{2}+m^{2}=1$ such that the polynomial equation

$$
a b\left(l^{2}+c m^{2}+X\right)^{4}-l^{4}\left(a l^{2}+c m^{2}+X\right)\left(b l^{2}+c m^{2}+X\right)=0
$$

has a real, strictly positive root for $X$. (Recall that we assume $\alpha, \beta, \gamma, \eta>0$.)

If $l=1$ and $m=0$, corresponding to a tangential wavenumber vector that is orthogonal to the unperturbed director field $\mathbf{n}_{0}$, then one can show that (62) has no real, positive root $X$ if

$$
\frac{1}{a}+\frac{1}{b} \leq 4
$$

We numerically computed the roots of (62) as $l$ and $m$ varied over $l^{2}+m^{2}=$ 1 , and found that if the parameters satisfy (63), then either $X<0$ or $X \in \mathbb{C}$ with $\Re X<0$. Although we do not have a proof, the numerical results suggest that the Lopatinski condition holds when (63) is satisfied (and $a, b, c>0$ ).

\subsection{Linearized surface waves}

Surface wave solutions correspond to Fourier-Laplace solutions with purely imaginary frequency $\tau=-i \omega_{0}$ with $\omega_{0} \in \mathbb{R}$ such that $L\left(-i \omega_{0}, l, m\right)=0$ for some $l, m \in \mathbb{R}$. It follows from (59) that the linearized surface-wave dispersion relation of $(54)-(55)$ for $\omega_{0}(l, m)$ is

$$
\left(\eta l^{2}+\gamma m^{2}-\omega_{0}^{2}\right)^{2}=\eta^{2} l^{2} \gamma_{\alpha} \gamma_{\beta}
$$


Table 2: Roots $X=x_{k}$ of the quartic polynomial (62) when $a=2, b=0.5$, and $c=3$ and $l^{2}+m^{2}=1$. Solutions with $X<0$ correspond to surface waves with speed $\lambda=(-\eta X)^{1 / 2}$

\begin{tabular}{|c||c|c|c|c|}
\hline$l$ & $x_{1}$ & $x_{2}$ & $x_{3}$ & $x_{4}$ \\
\hline \hline 0.000 & -3.0000 & -3.0000 & -3.0000 & -3.0000 \\
0.125 & $-2.9810+0.0082 \mathrm{i}$ & $-2.9810-0.0082 \mathrm{i}$ & -2.9599 & -2.9531 \\
0.250 & $-2.9239+0.0326 \mathrm{i}$ & $-2.9239-0.0326 \mathrm{i}$ & -2.8397 & -2.8125 \\
0.375 & $-2.8288+0.0734 \mathrm{i}$ & $-2.8288-0.0734 \mathrm{i}$ & -2.6393 & -2.5781 \\
0.500 & $-2.6956+0.1304 \mathrm{i}$ & $-2.6956-0.1304 \mathrm{i}$ & -2.3587 & -2.2500 \\
0.625 & $-2.5245+0.2038 \mathrm{i}$ & $-2.5245-0.2038 \mathrm{i}$ & -1.9980 & -1.8281 \\
0.750 & $-2.3152+0.2935 \mathrm{i}$ & $-2.3152-0.2935 \mathrm{i}$ & -1.5571 & -1.3125 \\
0.875 & $-2.0679+0.3994 \mathrm{i}$ & $-2.0679-0.3994 \mathrm{i}$ & -1.0360 & -0.7031 \\
1.000 & $-1.7826+0.5217 \mathrm{i}$ & $-1.7826-0.5217 \mathrm{i}$ & -0.4348 & 0 \\
\hline
\end{tabular}

where $\gamma_{\alpha}, \gamma_{\beta}>0$ are given by

$$
\gamma_{\alpha}=\sqrt{l^{2}+\frac{\gamma m^{2}-\omega_{0}^{2}}{\alpha}}, \quad \gamma_{\beta}=\sqrt{l^{2}+\frac{\gamma m^{2}-\omega_{0}^{2}}{\beta}} .
$$

It is convenient to introduce the scaled parameters (61). To find genuine surface wave solutions, we first look for real, negative roots $X=-\omega_{0}^{2} / \eta$ of (62). If such a root exists, then we check that $\gamma_{\alpha}, \gamma_{\beta}$ are real numbers. Finally, we verify that

$$
\left(l^{2}+c m^{2}+X\right)^{2}-l^{2} \sqrt{l^{2}+\frac{c m^{2}+X}{a}} \sqrt{l^{2}+\frac{c m^{2}+X}{b}}=0 .
$$

This last step is necessary to rule out extraneous solution due to squaring the Lopatinski condition to obtain (62).

There is a simple explicit solution of these equations with

$$
\omega_{0}^{2}=\gamma m^{2}, \quad \gamma_{\alpha}=\gamma_{\beta}=|l|,
$$

and

$$
\left[\begin{array}{l}
u \\
v
\end{array}\right]=\left[\begin{array}{c}
1 \\
i \operatorname{sgn} l
\end{array}\right] e^{i(l y+m z \pm \sqrt{\gamma} m t)-|l| x} .
$$

This is a genuine surface wave solution if $l \neq 0$. It turns out, however, that the quadratically nonlinear effects on this transverse surface wave vanish, so it is not of interest for the present analysis.

This is not the only solution, however. For example, in Table 2 we show the roots $X=x_{k}$ of (62) with $a=2, b=0.5, c=3$ for several values of $l$ 
Table 3: Decay constants for the negative root $x_{3}$ in Table 2, showing that it corresponds to a genuine surface wave

\begin{tabular}{|c||c|c|c|}
\hline$l$ & $x_{3}$ & $(\alpha / \eta)^{1 / 2} \gamma_{\alpha}$ & $(\beta / \eta)^{1 / 2} \gamma_{\beta}$ \\
\hline \hline 0.000 & -3.0000 & 0.0000 & 0.0000 \\
0.125 & -2.9599 & 0.0245 & 0.0010 \\
0.250 & -2.8397 & 0.0978 & 0.0041 \\
0.375 & -2.6393 & 0.2201 & 0.0092 \\
0.500 & -2.3587 & 0.3913 & 0.0163 \\
0.625 & -1.9980 & 0.6114 & 0.0255 \\
0.750 & -1.5571 & 0.8804 & 0.0367 \\
0.875 & -1.0360 & 1.1984 & 0.0499 \\
1.000 & -0.4348 & 1.5652 & 0.0652 \\
\hline
\end{tabular}

with $l^{2}+m^{2}=1$. The root $x_{4}$ corresponds to the explicit solution (66). The root $x_{3}$ is a second real, negative root. As Table 3 shows, it corresponds to a genuine surface wave.

In summary, surface wave solutions to the IBVP (54)-(55) can be written in the form

$$
\left[\begin{array}{l}
u \\
v
\end{array}\right]=\left\{R\left[\begin{array}{c}
i \gamma_{\alpha} \\
l
\end{array}\right] e^{-\gamma_{\alpha} x}+S\left[\begin{array}{c}
-l \\
i \gamma_{\beta}
\end{array}\right] e^{-\gamma_{\beta} x}\right\} e^{i\left(l y+m z-\omega_{0} t\right)} .
$$

Here, $\gamma_{\alpha}$ and $\gamma_{\beta}$ are given by (65), the surface-wave frequency $\omega_{0}$ satisfies

$$
\left(\eta l^{2}+\gamma m^{2}-\omega_{0}^{2}\right)^{2}-\eta^{2} l^{2} \sqrt{l^{2}+\frac{\gamma m^{2}-\omega_{0}^{2}}{\alpha}} \sqrt{l^{2}+\frac{\gamma m^{2}-\omega_{0}^{2}}{\beta}}=0,
$$

and $R, S$ satisfy (58). This solutions represents a genuine surface wave whose phase velocity along the boundary is at an angle $\phi=\tan ^{-1}(m / l)$ to $\mathbf{n}_{0}=$ $(0,0,1)$. General solutions may be obtained by Fourier-superposition of these solutions.

\subsection{Weakly nonlinear surface waves}

Asymptotic equations for weakly nonlinear surface wave solutions may derived following the procedure for the model problem. The algebra involved in a direct multiple-scale expansion is prohibitively complicated, but one can derive the equation by expanding the Hamiltonian or Lagrangian. Austria [5] gives details for the expansion of the Lagrangian. In this paper, we simply report the result. 
Let $\epsilon>0$ be a small dimensionless parameter. We introduce a "slow" time variable $\tau=\epsilon t$ and a "fast" phase variable

$$
\theta=l y+m z-\omega_{0} t
$$

where $(l, m) \in \mathbb{R}^{2}$ is a fixed nonzero vector, $\omega_{0}$ satisfies (64), and $\gamma_{\alpha}, \gamma_{\beta}>0$ are given in (65). The asymptotic solution has an expansion as $\epsilon \rightarrow 0$ of the form

$$
\mathbf{n}(x, \theta, \tau ; \epsilon)=\mathbf{n}_{0}+\epsilon \mathbf{n}_{1}(x, \theta, \tau)+O\left(\epsilon^{2}\right)
$$

where $\mathbf{n}_{0}=(0,0,1)$, and

$$
\mathbf{n}_{1}(x, \theta, \tau)=(u(x, \theta, \tau), v(x, \theta, \tau), 0)
$$

The first-order term $(u, v)$ is the linearized surface wave solution

$$
\begin{aligned}
{\left[\begin{array}{l}
u \\
v
\end{array}\right]=\int_{\mathbb{R}} \hat{a}(\rho, \tau) } & \left\{R(\rho)\left[\begin{array}{c}
i \operatorname{sgn}(\rho) \gamma_{\alpha} \\
l
\end{array}\right] e^{-\gamma_{\alpha}|\rho| x}\right. \\
& \left.+S(\rho)\left[\begin{array}{c}
l \\
-i \operatorname{sgn}(\rho) \gamma_{\beta}
\end{array}\right] e^{-\gamma_{\beta}|\rho| x}\right\} e^{i \rho \theta} d \rho
\end{aligned}
$$

where we represent the solution as a Fourier integral with respect to $\rho$, and

$$
R(\rho)=\eta l^{2}+\gamma m^{2}-\omega_{0}^{2}, \quad S(\rho)=-i \operatorname{sgn}(\rho) l \eta \gamma_{\alpha}
$$

Expanding the surface-wave Lagrangian up to cubic terms in $\hat{a}$, one finds that the corresponding Euler-Lagrange equation for $\hat{a}(\rho, \tau)$ has the form

$$
\hat{a}_{\tau}(\rho, \tau)+i \operatorname{sgn}(\rho) \int_{\mathbb{R}} \Lambda(-\rho, \rho-\xi, \xi) \hat{a}(\rho-\xi) \hat{a}(\xi) d \xi=0
$$

The kernel $\Lambda\left(k_{1}, k_{2}, k_{3}\right)$ in (70) is given on $k_{1}+k_{2}+k_{3}=0$ by

$$
\begin{aligned}
\Lambda\left(k_{1}, k_{2}, k_{3}\right) & =\frac{\Gamma_{0}\left(k_{1}, k_{2}, k_{3}\right)}{\left|k_{1}\right|+\left|k_{2}\right|+\left|k_{3}\right|} \\
& +\frac{\operatorname{sgn}\left(k_{2} k_{3}\right) \Gamma_{1}\left(k_{1}, k_{2}, k_{3}\right)}{\gamma_{\alpha}\left|k_{1}\right|+\gamma_{\beta}\left(\left|k_{2}\right|+\left|k_{3}\right|\right)}+\frac{\operatorname{sgn}\left(k_{1} k_{3}\right) \Gamma_{1}\left(k_{2}, k_{1}, k_{3}\right)}{\gamma_{\alpha}\left|k_{2}\right|+\gamma_{\beta}\left(\left|k_{1}\right|+\left|k_{3}\right|\right)} \\
& +\frac{\operatorname{sgn}\left(k_{1} k_{2}\right) \Gamma_{1}\left(k_{3}, k_{2}, k_{1}\right)}{\gamma_{\alpha}\left|k_{3}\right|+\gamma_{\beta}\left(\left|k_{1}\right|+\left|k_{2}\right|\right)}
\end{aligned}
$$




$$
\begin{aligned}
& +\frac{\operatorname{sgn}\left(k_{1}\right) \Gamma_{2}\left(k_{1}, k_{2}, k_{3}\right)}{\gamma_{\alpha}\left(\left|k_{2}\right|+\left|k_{3}\right|\right)+\gamma_{\beta}\left|k_{1}\right|}+\frac{\operatorname{sgn}\left(k_{2}\right) \Gamma_{2}\left(k_{2}, k_{1}, k_{3}\right)}{\gamma_{\alpha}\left(\left|k_{1}\right|+\left|k_{3}\right|\right)+\gamma_{\beta}\left|k_{2}\right|} \\
& +\frac{\operatorname{sgn}\left(k_{3}\right) \Gamma_{2}\left(k_{3}, k_{2}, k_{1}\right)}{\gamma_{\alpha}\left(\left|k_{1}\right|+\left|k_{2}\right|\right)+\gamma_{\beta}\left|k_{3}\right|},
\end{aligned}
$$

where $\Gamma_{0}, \Gamma_{1}, \Gamma_{2}$ are given by

$$
\begin{aligned}
& \quad \Gamma_{0}\left(k_{1}, k_{2}, k_{3}\right) \\
& =c_{1}(\gamma-\beta)\left(l^{2}-\gamma_{\beta}^{2}\right)\left[\left(k_{1}^{2}+k_{2}^{2}+k_{3}^{2}\right)-\left(\left|k_{1}\right|+\left|k_{2}\right|+\left|k_{3}\right|\right)^{2}\right] \\
& -c_{1}(\gamma-\beta)\left(l^{2}-\gamma_{\beta}^{2}\right)\left(k_{1}\left|k_{1}\right|+k_{2}\left|k_{2}\right|+k_{3}\left|k_{3}\right|\right) \operatorname{sgn}\left(k_{1} k_{2} k_{3}\right) \\
& \quad-c_{2}(\eta-\alpha)\left(l^{2}-\gamma_{\alpha}^{2}\right) \gamma_{\alpha}^{2}\left(k_{1}\left|k_{1}\right|+k_{2}\left|k_{2}\right|+k_{3}\left|k_{3}\right|\right) \operatorname{sgn}\left(k_{1} k_{2} k_{3}\right) \\
& \quad+c_{2}(\eta-\alpha)\left(l^{2}-\gamma_{\alpha}^{2}\right) l^{2}\left(k_{1}^{2}+k_{2}^{2}+k_{3}^{2}\right) \\
& \quad-c_{1}(\gamma-\eta) \gamma_{\beta}^{2}\left(\left|k_{1}\right|+\left|k_{2}\right|+\left|k_{3}\right|\right)^{2} \\
& \quad+c_{2}(\gamma-\eta) \gamma_{\alpha}^{2} l^{2}\left(\left|k_{1}\right|+\left|k_{2}\right|+\left|k_{3}\right|\right)^{2}, \\
& \Gamma_{1}\left(k_{1}, k_{2}, k_{3}\right) \\
& =c_{3}(\gamma-\beta)\left(l^{2}-\gamma_{\beta}^{2}\right)\left(l^{2}-\operatorname{sgn}\left(k_{1} k_{2}\right) \gamma_{\alpha} \gamma_{\beta}\right)\left(k_{1}^{2}-k_{2}^{2}\right) \\
& +c_{3}(\gamma-\beta)\left(l^{2}-\gamma_{\beta}^{2}\right)\left(l^{2}-\operatorname{sgn}\left(k_{1} k_{3}\right) \gamma_{\alpha} \gamma_{\beta}\right)\left(k_{1}^{2}-k_{3}^{2}\right) \\
& -c_{3}(\eta-\alpha)\left(l^{2}-\gamma_{\alpha}^{2}\right)\left(l^{2}-\operatorname{sgn}\left(k_{2} k_{3}\right) \gamma_{\beta}^{2}\right) k_{1}^{2} \\
& -c_{3}(\gamma-\eta) l^{2}\left(\operatorname{sgn}\left(k_{1}\right) \gamma_{\alpha}-\operatorname{sgn}\left(k_{3}\right) \gamma_{\beta}\right)\left(\gamma_{\alpha}\left|k_{1}\right|+\gamma_{\beta}\left(\left|k_{2}\right|+\left|k_{3}\right|\right)\right) k_{2} \\
& -c_{3}(\gamma-\eta) l^{2}\left(\operatorname{sgn}\left(k_{1}\right) \gamma_{\alpha}-\operatorname{sgn}\left(k_{2}\right) \gamma_{\beta}\right)\left(\gamma_{\alpha}\left|k_{1}\right|+\gamma_{\beta}\left(\left|k_{2}\right|+\left|k_{3}\right|\right)\right) k_{3} \\
& -c_{3}(\gamma-\eta)\left(l^{2}-\operatorname{sgn}\left(k_{2} k_{3}\right) \gamma_{\beta}^{2}\right)\left(\gamma_{\alpha} \gamma_{\beta}\left(\left|k_{1} k_{2}\right|+\left|k_{1} k_{3}\right|\right)+l^{2} k_{1}^{2}\right),
\end{aligned}
$$

$$
\begin{aligned}
& \Gamma_{2}\left(k_{1}, k_{2}, k_{3}\right) \\
& \quad=c_{4}(\gamma-\beta)\left(l^{2}-\gamma_{\beta}^{2}\right) \gamma_{\alpha}\left(\operatorname{sgn}\left(k_{3}\right)-\operatorname{sgn}\left(k_{2}\right)\right)\left(k_{3}^{2}-k_{2}^{2}\right) \\
& \quad-c_{4}(\eta-\alpha)\left(l^{2}-\gamma_{\alpha}^{2}\right)\left(\operatorname{sgn}\left(k_{1}\right) \gamma_{\beta}-\operatorname{sgn}\left(k_{3}\right) \gamma_{\alpha}\right) k_{2}^{2} \\
& \quad-c_{4}(\eta-\alpha)\left(l^{2}-\gamma_{\alpha}^{2}\right)\left(\operatorname{sgn}\left(k_{1}\right) \gamma_{\beta}-\operatorname{sgn}\left(k_{2}\right) \gamma_{\alpha}\right) k_{3}^{2} \\
& -c_{4}(\gamma-\eta)\left(\operatorname{sgn}\left(k_{1}\right) \gamma_{\beta}-\operatorname{sgn}\left(k_{3}\right) \gamma_{\alpha}\right)\left(\gamma_{\alpha}^{2}\left|k_{2} k_{3}\right|+\gamma_{\alpha} \gamma_{\beta}\left|k_{1} k_{2}\right|+l^{2} k_{2}^{2}\right) \\
& -c_{4}(\gamma-\eta)\left(\operatorname{sgn}\left(k_{1}\right) \gamma_{\beta}-\operatorname{sgn}\left(k_{2}\right) \gamma_{\alpha}\right)\left(\gamma_{\alpha}^{2}\left|k_{2} k_{3}\right|+\gamma_{\alpha} \gamma_{\beta}\left|k_{1} k_{3}\right|+l^{2} k_{3}^{2}\right) \\
& \quad-c_{4}(\gamma-\eta)\left(l^{2}-\operatorname{sgn}\left(k_{2} k_{3}\right) \gamma_{\alpha}^{2}\right)\left(\gamma_{\alpha}\left(\left|k_{2}\right|+\left|k_{3}\right|\right)+\gamma_{\beta}\left|k_{1}\right|\right) k_{1} .
\end{aligned}
$$

The constants are given by

$$
c_{1}=\gamma_{\alpha}\left[\frac{m\left(\eta l \gamma_{\alpha}\right)^{2} \eta l^{2}}{4 E_{0}}\right]
$$




$$
\begin{aligned}
& c_{2}=\frac{\gamma_{\beta}}{\gamma_{\alpha}^{2}}\left[\frac{m\left(\eta l \gamma_{\alpha}\right)^{2}}{4 E_{0}}\right]\left(\eta l^{2}+\gamma m^{2}-\omega_{0}^{2}\right) \\
& c_{3}=\left[\frac{m\left(\eta l \gamma_{\alpha}\right)^{2}}{4 E_{0}}\right]\left(\eta l^{2}+\gamma m^{2}-\omega_{0}^{2}\right) \\
& c_{4}=\gamma_{\beta}\left[\frac{m\left(\eta l \gamma_{\alpha}\right)^{2} \eta l^{2}}{4 E_{0}}\right],
\end{aligned}
$$

where

$$
E_{0}=\left(\frac{l^{2}+\gamma_{\alpha}^{2}}{2 \gamma_{\alpha}}\right)|R|^{2}+\left(\frac{l^{2}+\gamma_{\beta}^{2}}{2 \gamma_{\beta}}\right)|S|^{2}-i l\left[R S^{*}-R^{*} S\right]
$$

We note that for the surface wave solution (66), we get

$$
\begin{aligned}
\Gamma_{0}\left(k_{1}, k_{2}, k_{3}\right) & =(\gamma-\eta) l^{2}\left(c_{2} l^{2}-c_{1}\right)\left(\left|k_{1}\right|+\left|k_{2}\right|+\left|k_{3}\right|\right)^{2}, \\
\operatorname{sgn}\left(k_{2} k_{3}\right) \Gamma_{1}\left(k_{1}, k_{2}, k_{3}\right) & =(\gamma-\eta) l^{2}\left(c_{3} l\right)\left(\left|k_{1}\right|+\left|k_{2}\right|+\left|k_{3}\right|\right)^{2}, \\
\operatorname{sgn}\left(k_{1}\right) \Gamma_{2}\left(k_{1}, k_{2}, k_{3}\right) & =(\gamma-\eta) l^{2}\left(c_{4}\right)\left(\left|k_{1}\right|+\left|k_{2}\right|+\left|k_{3}\right|\right)^{2},
\end{aligned}
$$

and $3\left(c_{3} l-c_{4}\right)+c_{2} l^{2}-c_{1}=0$. Thus, $\Lambda\left(k_{1}, k_{2}, k_{3}\right)=0$ for all $k_{1}, k_{2}, k_{3}$ with $k_{1}+k_{2}+k_{3}=0$, so the quadratically nonlinear effects drop out, and one would have to include higher-order terms in the expansion (67) to determine the nonlinear behavior of these surface waves.

If $\omega_{0}^{2} \neq \gamma m^{2}$, then the asymptotic equation is typically nontrivial, and we get a qualitatively similar kernel to the one we obtained for the model equation.

\section{Appendix}

In this Appendix, we briefly describe the derivation of the multiple-scale solution (23) of (9)-(10) for a weakly nonlinear surface wave. Further details of the computations are given in [5].

We introduce multiple-scale variables $\theta=y-\lambda t, x, \tau=\epsilon t$ and expand partial derivatives with respect to $x, y, t$ as

$$
\partial_{x}=\partial_{x}, \quad \partial_{y}=\partial_{\theta}, \quad \partial_{t}=-\lambda \partial_{\theta}+\epsilon \partial_{\tau}
$$

We use these expansions and (22)-(23) in (9)-(10) and equate coefficients of $\epsilon$ and $\epsilon^{2}$ to zero in the resulting equations. 
At the order $\epsilon$, we find that $\left(u_{1}, v_{1}\right)$ satisfies

$$
\left(\alpha_{0}^{2}-\lambda^{2}\right) u_{1 \theta \theta}+\alpha_{0}^{2} u_{1 x x}=0, \quad\left(\beta_{0}^{2}-\lambda^{2}\right) v_{1 \theta \theta}+\beta_{0}^{2} v_{1 x x}=0
$$

in $x>0$, with the boundary condition

$$
\alpha_{0}^{2} u_{1 x}-\eta v_{1 \theta}=0, \quad \beta_{0}^{2} v_{1 x}+\eta u_{1 \theta}=0
$$

on $x=0$. The slow time $\tau$ occurs as a parameter in these equations.

At the order $\epsilon^{2}$, we find that $\left(u_{2}, v_{2}\right)$ satisfies

$$
\begin{gathered}
\left(\alpha_{0}^{2}-\lambda^{2}\right) u_{2 \theta \theta}+\alpha_{0}^{2} u_{2 x x}=-2 \lambda u_{1 \theta \tau} \\
+\alpha_{0} \alpha_{1}\left[u_{1 x}^{2}-\left(u_{1}^{2}\right)_{x x}+u_{1 \theta}^{2}-\left(u_{1}^{2}\right)_{\theta \theta}\right] \\
\left(\beta_{0}^{2}-\lambda^{2}\right) v_{2 \theta \theta}+\beta_{0}^{2} v_{2 x x}=-2 \lambda v_{1 \theta \tau} \\
+\beta_{0} \beta_{1}\left[v_{1 x}^{2}-\left(v_{1}^{2}\right)_{x x}+v_{1 \theta}^{2}-\left(v_{1}^{2}\right)_{\theta \theta}\right]
\end{gathered}
$$

in $x>0$, with the boundary condition

$$
\begin{aligned}
& \alpha_{0}^{2} u_{2 x}-\eta v_{2 \theta}=-\alpha_{0} \alpha_{1}\left(u_{1}^{2}\right)_{x}, \\
& \beta_{0}^{2} v_{2 x}+\eta u_{2 \theta}=-\beta_{0} \beta_{1}\left(v_{1}^{2}\right)_{x}
\end{aligned}
$$

on $x=0$.

The Fourier-Laplace solution of the leading-order equations (75)-(76) has the form

$$
\left[\begin{array}{l}
u_{1} \\
v_{1}
\end{array}\right](x, \theta, \tau)=\int_{\mathbb{R}} \hat{a}(k, \tau)\left[\begin{array}{c}
R(k) e^{-\gamma_{\alpha}|k| x} \\
S(k) e^{-\gamma_{\beta}|k| x}
\end{array}\right] e^{i k \theta} d k,
$$

where $\hat{a}(-k, \tau)=\hat{a}^{*}(-k, \tau)$. This expression satisfies (75) if

$$
\gamma_{\alpha}=\sqrt{1-\frac{\lambda^{2}}{\alpha_{0}^{2}}}, \quad \gamma_{\beta}=\sqrt{1-\frac{\lambda^{2}}{\beta_{0}^{2}}},
$$

and it satisfies (76) if

$$
\left[\begin{array}{cc}
-\alpha_{0}^{2} \gamma_{\alpha}|k| & -i \eta k \\
i \eta k & -\beta_{0}^{2} \gamma_{\beta}|k|
\end{array}\right]\left[\begin{array}{c}
R(k) \\
S(k)
\end{array}\right]=\left[\begin{array}{l}
0 \\
0
\end{array}\right] .
$$

Equation (80) has a nontrivial solution for $(R, S)$ if $\alpha_{0}^{2} \beta_{0}^{2} \gamma_{\alpha} \gamma_{\beta}=\eta^{2}$, which implies that 


$$
\lambda^{2}=\frac{1}{2}\left\{\alpha_{0}^{2}+\beta_{0}^{2} \pm \sqrt{\left(\alpha_{0}^{2}-\beta_{0}^{2}\right)^{2}+\frac{4 \eta^{4}}{\alpha_{0}^{2} \beta_{0}^{2}}}\right\} .
$$

We choose the negative sign, as in (27), in which case $\gamma_{\alpha}$ and $\gamma_{\beta}$ are real and positive for $\alpha_{0} \beta_{0}>|\eta|$. We also take $R=\eta$ and $S=i \operatorname{sgn}(k) \alpha_{0}^{2} \gamma_{\alpha}$.

Next, using the Fourier-Laplace transform, we solve the non-homogeneous system of PDEs $(77)$ for $\left(u_{2}, v_{2}\right)$, where $\left(u_{1}, v_{1}\right)$ is given by (79). After some algebra, we find that

$$
\begin{aligned}
u_{2} & =\int_{\mathbb{R}}\left[R_{0}(k)+i \operatorname{sgn}(k) \frac{\eta}{\alpha_{0}^{2} \gamma_{\alpha}} \lambda \hat{a}_{\tau}(k, \tau) x\right] e^{i k \theta-\gamma_{\alpha}|k| x} d k \\
& +\iint_{U_{1}} R_{1}(k, l) \hat{a}(k, \tau) \hat{a}(l, \tau) x e^{i(k+l) \theta-\gamma_{\alpha}(|k|+|l|) x} d k d l \\
& +\iint_{U_{2}} R_{2}(k, l) \hat{a}(k, \tau) \hat{a}(l, \tau) e^{i(k+l) \theta-\gamma_{\alpha}(|k|+|l|) x} d k d l, \\
v_{2} & \left.=\int S_{\mathbb{R}} S_{0}(k)-\frac{\alpha_{0}^{2} \gamma_{\alpha}}{\beta_{0}^{2} \gamma_{\beta}} \lambda \hat{a}_{\tau}(k, \tau) x\right] e^{i k \theta-\gamma_{\beta}|k| x} d k \\
& +\iint_{U_{1}} S_{1}(k, l) \hat{a}(k, \tau) \hat{a}(l, \tau) x e^{i(k+l) \theta-\gamma_{\beta}(|k|+|l|) x} d k d l \\
& +\iint_{U_{2}} S_{2}(k, l) \hat{a}(k, \tau) \hat{a}(l, \tau) e^{i(k+l) \theta-\gamma_{\beta}(|k|+|l|) x} d k d l,
\end{aligned}
$$

where $U_{1}=\left\{(l, k) \in \mathbb{R}^{2}: \operatorname{sgn} k=\operatorname{sgn} l\right\}, U_{2}=\left\{(k, l) \in \mathbb{R}^{2}: \operatorname{sgn} k \neq \operatorname{sgn} l\right\}$,

$$
\begin{aligned}
& R_{1}(k, l)=\frac{\alpha_{0} \alpha_{1} \eta^{2}\left[\gamma_{\alpha}^{2}\left((|k|+|l|)^{2}-|k||l|\right)-\left((k+l)^{2}-k l\right)\right]}{2 \alpha_{0}^{2} \gamma_{\alpha}(|k|+|l|)} \\
& R_{2}(k, l)=-\frac{\alpha_{0} \alpha_{1} \eta^{2}\left[\gamma_{\alpha}^{2}\left((|k|+|l|)^{2}-|k||l|\right)-\left((k+l)^{2}-k l\right)\right]}{\alpha_{0}^{2} \gamma_{\alpha}^{2}\left[(|k|+|1|)^{2}-(k+l)^{2}\right]} \\
& S_{1}(k, l)=-\frac{\beta_{0} \beta_{1}\left(\alpha_{0}^{2} \gamma_{\alpha}\right)^{2} \operatorname{sgn}(k) \operatorname{sgn}(l)\left[\gamma_{\beta}^{2}\left((|k|+|l|)^{2}-|k||l|\right)-\left((k+l)^{2}-k l\right)\right]}{2 \beta_{0}^{2} \gamma_{\beta}(|k|+|l|)} \\
& S_{2}(k, l)=\frac{\beta_{0} \beta_{1}\left(\alpha_{0}^{2} \gamma_{\alpha}\right)^{2} \operatorname{sgn}(k) \operatorname{sgn}(l)\left[\gamma_{\beta}^{2}\left((|k|+|l|)^{2}-|k||l|\right)-\left((k+l)^{2}-k l\right)\right]}{\beta_{0}^{2} \gamma_{\beta}^{2}\left[(|k|+|l|)^{2}-(k+l)^{2}\right]},
\end{aligned}
$$

and $R_{0}(k), S_{0}(k)$ are arbitrary functions of integration.

Using (79) and (81) in the second-order boundary condition (78) and simplifying the result, we get a singular linear system of equations for 
$\left(R_{0}, S_{0}\right)$ of the form

$$
\left[\begin{array}{cc}
-\alpha_{0}^{2} \gamma_{\alpha}|k| & -i \eta k \\
i \eta k & -\beta_{0}^{2} \gamma_{\beta}|k|
\end{array}\right]\left[\begin{array}{c}
R_{0}(k) \\
S_{0}(k)
\end{array}\right]=\left[\begin{array}{c}
F_{1} \\
F_{2}
\end{array}\right],
$$

where

$$
\begin{aligned}
& F_{1}=-i \operatorname{sgn}(k) \eta \lambda \hat{a}_{\tau}(k, \tau)+\int_{-\infty}^{\infty} \Gamma_{1}(k-l, l) \hat{a}(k-l, \tau) \hat{a}(l, \tau) d l \\
& F_{2}=\frac{\alpha_{0}^{2} \gamma_{\alpha}}{\gamma_{\beta}} \lambda \hat{a}_{\tau}(k, \tau)+\int_{-\infty}^{\infty} \Gamma_{2}(k-l, l) \hat{a}(k-l, \tau) \hat{a}(l, \tau) d l .
\end{aligned}
$$

The kernels $\Gamma_{i}(k-l, l)$ in $F_{i}$ are given by

$$
\Gamma_{1}(k-l, l)= \begin{cases}P_{2}(k-l, l), & \text { if } k>0 \text { and }-\infty<l<0 \\ P_{1}(k-l, l), & \text { if } k>0 \text { and } 0<l<k \\ P_{2}(k-l, l), & \text { if } k>0 \text { and } k<l<+\infty \\ P_{2}(k-l, l), & \text { if } k<0 \text { and }-\infty<l<k \\ P_{1}(k-l, l), & \text { if } k<0 \text { and } k<l<0 \\ P_{2}(k-l, l), & \text { if } k<0 \text { and } 0<l<+\infty\end{cases}
$$

and

$$
\Gamma_{2}(k-l, l)= \begin{cases}Q_{2}(k-l, l), & \text { if } k>0 \text { and }-\infty<l<0 \\ Q_{1}(k-l, l), & \text { if } k>0 \text { and } 0<l<k \\ Q_{2}(k-l, l), & \text { if } k>0 \text { and } k<l<+\infty \\ Q_{2}(k-l, l), & \text { if } k<0 \text { and }-\infty<l<k \\ Q_{1}(k-l, l), & \text { if } k<0 \text { and } k<l<0 \\ Q_{2}(k-l, l), & \text { if } k<0 \text { and } 0<l<+\infty\end{cases}
$$

where

$$
\begin{aligned}
P_{1}(k, l) & =\frac{\alpha_{0} \alpha_{1} \gamma_{\alpha} \eta^{2}}{2}\left[(|k|+|l|)^{2}+|k||l|+\frac{(k+l)^{2}-k l}{\gamma_{\alpha}^{2}}\right] \frac{1}{|k|+|l|} \\
P_{2}(k, l) & =\frac{\alpha_{0} \alpha_{1} \gamma_{\alpha} \eta^{2}}{2}\left[|k||l|-(k+l)^{2}+\frac{(k+l)^{2}-k l}{\gamma_{\alpha}^{2}}\right] \frac{|k|+|l|}{|k||l|-k l} \\
& +i \frac{\beta_{1}\left(\alpha_{0}^{2} \gamma_{\alpha}\right)^{2} \eta}{2 \beta_{0}}\left[(|k|+|l|)^{2}-|k||l|-\frac{(k+l)^{2}-k l}{\gamma_{\beta}^{2}}\right] \\
& \times \frac{(k+l) \operatorname{sgn}(k) \operatorname{sgn}(k)}{|k||l|-k l}
\end{aligned}
$$


and

$$
\begin{aligned}
Q_{1}(k, l) & =-\frac{\beta_{0} \beta_{1} \gamma_{\beta}\left(\alpha_{0}^{2} \gamma_{\alpha}\right)^{2}}{2}\left[(|k|+|l|)^{2}+|k||l|+\frac{(k+l)^{2}-k l}{\gamma_{\beta}^{2}}\right] \frac{\operatorname{sgn}(k) \operatorname{sgn}(l)}{|k|+|l|} \\
Q_{2}(k, l) & =-\frac{\beta_{0} \beta_{1} \gamma_{\beta}\left(\alpha_{0}^{2} \gamma_{\alpha}\right)^{2}}{2}\left[|k||l|-(k+l)^{2}+\frac{\left.(k+l)^{2}-k l\right]}{\gamma_{\beta}^{2}}\right] \\
& \times \frac{(|k|+|l|) \operatorname{sgn}(k) \operatorname{sgn}(l)}{|k||l|-k l} \\
& +i \frac{\alpha_{1} \eta^{3}}{2 \alpha_{0}}\left[(|k|+|l|)^{2}-|k||l|-\frac{\left.(k+l)^{2}-k l\right]}{\gamma_{\alpha}^{2}}\right] \frac{(k+l)}{|k||l|-k l} .
\end{aligned}
$$

The solvability condition for $(82)$ is

$$
\eta F_{1}-i \operatorname{sgn}(k) \alpha_{0}^{2} \gamma_{\alpha} F_{2}=0
$$

Using (83) in this condition and simplifying the result, we get an equation for $\hat{a}(k, \tau)$, which may be written as

$$
\hat{a}_{\tau}(k, \tau)+i \operatorname{sgn}(k) \int_{-\infty}^{+\infty} \Lambda(-k, k-l, l) \hat{a}(k-l, \tau) \hat{a}(l, \tau) d l=0,
$$

where

$$
\Lambda(-k, k-l, l)=\frac{\gamma_{\alpha} \gamma_{\beta} \eta^{2}}{\lambda\left(\alpha_{0}^{2} \gamma_{\alpha}^{2}+\beta_{0}^{2} \gamma_{\beta}^{2}\right)}\left[\frac{1}{\alpha_{0}^{2} \gamma_{\alpha} \eta} \Gamma_{1}(k-l, l)-\frac{i \operatorname{sgn}(k)}{\eta^{2}} \Gamma_{2}(k-l, l)\right] .
$$

Finally, by considering the possible signs of $-k, k-l, l$ one can verify that this expression for $\Lambda(-k, k-l, l)$ agrees with the expression given in (12) for $\Lambda\left(k_{1}, k_{2}, k_{3}\right)$ on $k_{1}+k_{2}+k_{3}=0$.

\section{References}

[1] G. Alì and J. K. Hunter, Nonlinear surface waves on a tangential discontinuity in magnetohydrodynamics, Quart. Appl. Math., 61 (2003), 451-474. MR1999831

[2] G. Alì and J. K. Hunter, Orientation waves in a director field with rotational inertia, Kinet. Relat. Models, 2 (2009), 1-37. MR2472148

[3] G. Alì, J. K. Hunter, and D. F. Parker, Hamiltonian Equations for ScaleInvariant Waves, Stud. Appl. Math., 108 (2002), 305-321. MR1895286 
[4] M. Artola and A. J. Majda, Nonlinear development of instabilities in supersonic vortex sheets I, The basic kink modes, Physica D, 28 (1987), 253-281. MR0914450

[5] L. Austria, Nonlinear Hyperbolic Surface Waves in Variational Boundary-Value Problems, PhD thesis, University of California at Davis, 2011. MR2982248

[6] S. Benzoni-Gavage and D. Serre, Multidimensional Hyperbolic Partial Differential Equations: First-Order Systems and Applications, Clarendon Press, 2007. MR2284507

[7] A. Bressan and A. Constantin, Global solutions of the Hunter-Saxton equation, SIAM J. Math. Anal., 37 (2005), 996-1026. MR2191785

[8] A. Bressan and Y. Zheng, Conservative Solutions to a Nonlinear Variational Wave Equation, Commun. Math. Phys., 266 (2006), 471-497. MR2238886

[9] A. Bressan, P. Zhang, and Y. Zheng, Asymptotic variational wave equations, Arch. Ration. Mech. Anal., 183 (2007), 163-185. MR2259342

[10] G. Chen, P. Zhang, and Y. Zheng, Energy conservative solutions to a nonlinear wave system of nematic liquid crystals, Commun. Pure Appl. Anal., 12 (2013), 1445-1468. MR2989699

[11] C. M. Dafermos, Hyperbolic Conservation Laws in Continuum Physics, 2nd ed., Springer, 2005. MR2169977

[12] P.-G. de Gennes and J. Prost, The Physics of Liquid Crystals, Clarendon Press, 1995.

[13] W. Domański and A. N. Norris, Degenerate nonlinear elastic plane waves, Int. J. Non-Linear Mech., 44 (2009), 486-493.

[14] Y. Du, G. Chen, and J. Liu, The almost global existence to classical solutions for a 3-d wave equation of nematic liquid crystals, preprint.

[15] M. F. Hamilton, Y. A. Il'insky, and E. A. Zabolotskaya, Evolution equations for nonlinear Rayleigh waves, J. Acoustical Society of America, 97 (1995), 891-897.

[16] J. K. Hunter, Nonlinear Surface Waves, Contemporary Mathematics, 100 (1989), 185-202. MR1033516

[17] J. K. Hunter, Short-Time Existence for Scale-Invariant Hamiltonian Waves, J. Hyperbolic Differ. Equ., 3 (2006), 247-265. MR2229856 
[18] J. K. Hunter, Nonlinear surface waves, in IMA Volume on Nonlinear Conservation Laws and Applications, eds. A. Bressan et al., 303-314, Springer, 2011. MR2857003

[19] J. K. Hunter and R. Saxton, Dynamics of Director Fields, SIAM J. Appl. Math., 51 (1991), 1498-1521. MR1135995

[20] J. K. Hunter and J. B. Thoo, On the weakly nonlinear Kelvin-Helmholtz instability of tangential discontinuities in MHD, J. Hyperbolic Differ. Equ., 8 (2011), 691-726. MR2864545

[21] J. K. Hunter and Y. Zheng, On a completely integrable nonlinear hyperbolic variational equation, Physica D, 79 (1994), 361-386. MR1306466

[22] J. K. Hunter and Y. Zheng, On a nonlinear hyperbolic variational equation I and II, Arch. Ration. Mech. Anal., 129 (1995), 305-353, 355-383.

[23] H.-O. Kreiss, Initial boundary value problems for hyperbolic systems, Comm. Pure Appl. Math., 23 (1970), 277-298. MR0437941

[24] A. J. Majda, Compressible Fluid Flow and Systems of Conservation Laws, Springer-Verlag, New York, 1984.

[25] R. Sakamoto, Mixed problems for hyperbolic equations I. Energy inequalities, J. Math. Kyoto Univ., 10 (1970), 349-373. MR0283400

[26] R. Sakamoto, Mixed problems for hyperbolic equations II. Existence theorems with zero initial datas and energy inequalities with initial datas, J. Math. Kyoto Univ., 10 (1970), 403-417. MR0283401

[27] D. Serre, Second order initial boundary-value problems of variational type, J. Funct. Anal., 236 (2006), 409-446. MR2240169

[28] R. Temam, Infinite Dimensonal Dynamical Systems in Mechanics and Physics, 2nd ed., Springer, 1997. MR1441312

[29] E. G. Virga, Variational Theories for Liquid Crystals, Chapman \& Hall, 1994. MR1369095

[30] V. E. Zakharov and E. A. Kuznetsov, Hamiltonian formalism for nonlinear waves, Usp. Fiz. Nauk., 167 (1997), 1137-1168.

[31] P. Zhang and Y. Zheng, Existence and uniqueness of solutions to an asymptotic equation of a variational wave equation, Arch. Ration. Mech. Anal., 155 (2000), 49-83. MR1799274

[32] P. Zhang and Y. Zheng, Energy conservative solutions to a onedimensional full variational wave system, Comm. Pure Appl. Math., 65 (2012), 683-726. MR2898888 
LaWrence Austria

Naval Air Warfare Center

AIRCRAFT Division

PAtuxent River, MD 20670

USA

E-mail address: lawrence.austria@navy.mil

JoHn K. Hunter

Department of Mathematics

University of CALIForNia at Davis

DAVIS, CA 95616

USA

E-mail address: jkhunter@ucdavis.edu

Received September 9, 2013 